\title{
LARGE DEVIATIONS FOR SYMMETRIC STABLE PROCESSES WITH FEYNMAN-KAC FUNCTIONALS AND ITS APPLICATION TO PINNED POLYMERS
}

\author{
YASUHITO NISHIMORI
}

(Received October 9, 2012, revised January 28, 2013)

\begin{abstract}
Let $v$ and $\mu$ be positive Radon measures on $\boldsymbol{R}^{d}$ in Green-tight Kato class associated with a symmetric $\alpha$-stable process $\left(X_{t}, P_{x}\right)$ on $\boldsymbol{R}^{d}$, and $A_{t}^{\nu}$ and $A_{t}^{\mu}$ the positive continuous additive functionals under the Revuz correspondence to $v$ and $\mu$. For a nonnegative $\beta$, let $P_{x, t}^{\beta \mu}$ be the law $X_{t}$ weighted by the Feynman-Kac functional $\exp \left(\beta A_{t}^{\mu}\right)$, i.e., $P_{x, t}^{\mu}=\left(Z_{x, t}^{\mu}\right)^{-1} \exp \left(\beta A_{t}^{\mu}\right) P_{x}$, where $Z_{x, t}^{\mu}$ is a normalizing constant. We show that $A_{t}^{v} / t$ obeys the large deviation principle under $P_{x, t}^{\beta \mu}$. We apply it to a polymer model to identify the critical value $\beta_{\mathrm{cr}}$ such that the polymer is pinned under the law $P_{x, t}^{\beta \mu}$ if and only if $\beta$ is greater than $\beta_{\mathrm{cr}}$. The value $\beta_{\mathrm{cr}}$ is characterized by the rate function.
\end{abstract}

1. Introduction. We consider a homopolymer consisting of a lot of monomers. Mathematically, the homopolymer can be modeled by a path of Brownian motion. Let $\left(\left\{B_{t}\right\}_{t \geq 0}, P_{0}\right)$ be the 1-dimensional Brownian motion with starting at the origin and, for $\beta \geq 0$, define

$$
\begin{gathered}
P_{0, t}^{\beta \delta_{0}}(d \omega)=\left(Z_{0, t}^{\beta \delta_{0}}\right)^{-1} \exp \left(\beta \int_{0}^{t} \delta_{0}\left(B_{s}(\omega)\right) d s\right) P_{0}(d \omega), \\
Z_{0, t}^{\beta \delta_{0}}=E_{0}\left[\exp \left(\beta \int_{0}^{t} \delta_{0}\left(B_{s}\right) d s\right)\right] .
\end{gathered}
$$

Here $\beta$ means the inverse temperature. The local time at the origin, $\int_{0}^{t} \delta_{0}\left(B_{S}\right) d s$ is regarded as the energy of the polymer. The polymer is said to be pinned if there exists some $\kappa>0$ such that

$$
\lim _{t \rightarrow \infty} P_{0, t}^{\beta \delta_{0}}\left(\frac{\int_{0}^{t} \delta_{0}\left(B_{s}\right) d s}{t}>\kappa\right)=1 .
$$

This notion is introduced by Alexander and Sidoravicius [2]. When the underlying process is the simple random walk on the integer lattice $\boldsymbol{Z}^{d}$, Cranston and Molchanov [7] proved that if $d=1,2$, then for every $\beta>0$ there exists such $\kappa>0$ and if $d \geq 3$, then there exists a critical temperature $\beta_{\mathrm{cr}}>0$ such that for any $\beta>\beta_{\mathrm{cr}}$, there exists $\kappa>0$ satisfying the equation (1.3). Our objective is to calculate the supremum of $\kappa$ satisfying (1.3) and to estimate the order of convergence of the limit (1.3). In addition, we would like to extend the underlying

2000 Mathematics Subject Classification. Primary 60F10; Secondary 82D60, 60G52.

Key words and phrases. Pinned polymer, large deviations, Dirichlet form, symmetric stable process, additive functional. 
process to symmetric $\alpha$-stable processes, i.e., Markov processes generated by the fractional Laplacian $(-\Delta)^{\alpha / 2}, 0<\alpha<2$.

For the proof of the equality (1.3), we see that the large deviation principle (LDP) for the local time of the Brownian motion with respect to the probability measure $P_{0, t}^{\beta \delta_{0}}$ is applicable. Hence we first prove the LDP for additive functionals of symmetric $\alpha$-stable processes weighted by Feynman-Kac functionals. In fact, the local time is a typical additive functional corresponding to the Dirac measure. It is proper to realize the energy as an additive functional. More precisely, for a positive Green-tight Kato measure $\mu$ on $\boldsymbol{R}^{d}$ ( $\mu \in \mathcal{K}_{d, \alpha}^{\infty}$ in notation), we define the energy of polymer by the positive continuous additive functionals (PCAF) $A_{t}^{\mu}$ under the Revuz correspondence to $\mu$. Let us denote

$$
P_{x, t}^{\mu}(d \omega)=\left(Z_{x, t}^{\mu}\right)^{-1} \exp \left(A_{t}^{\mu}(\omega)\right) P_{x}(d \omega),
$$

where $Z_{x, t}^{\mu}$ is the normalizing factor, $Z_{x, t}^{\mu}=E_{x}\left[\exp \left(A_{t}^{\mu}\right)\right]$. $Z_{x, t}^{\mu}$ is called a partition function. We define the logarithmic moment generating function $C_{N}(\lambda)$ of $A_{t}^{\nu}$ under $P_{x, t}^{\mu}$ by

$$
C_{N}(\lambda)=\lim _{t \rightarrow \infty} \frac{1}{t} \log E_{x, t}^{\mu}\left[\exp \left(\lambda A_{t}^{\nu}\right)\right], \quad \lambda \in \boldsymbol{R},
$$

where $E_{x, t}^{\mu}[\cdot]$ is the expectation under the probability measure $P_{x, t}^{\mu} . C_{N}^{*}(z)$ is the FenchelLegendre transformation of $C_{N}(\lambda)$, i.e.,

$$
C_{N}^{*}(z)=\sup _{\lambda \in \boldsymbol{R}}\left(z \lambda-C_{N}(\lambda)\right), \quad z \in \boldsymbol{R} .
$$

We then have the next theorem.

THEOREM 1.1. Let $v, \mu \in \mathcal{K}_{d, \alpha}^{\infty}(\alpha<d \leq 2 \alpha)$.

(i) For any open set $G \subset \boldsymbol{R}$,

$$
\liminf _{t \rightarrow \infty} \frac{1}{t} \log P_{x, t}^{\mu}\left(\frac{A_{t}^{\nu}}{t} \in G\right) \geq-\inf _{z \in G} C_{N}^{*}(z) .
$$

(ii) For any closed set $F \subset \boldsymbol{R}$,

$$
\limsup _{t \rightarrow \infty} \frac{1}{t} \log P_{x, t}^{\mu}\left(\frac{A_{t}^{v}}{t} \in F\right) \leq-\inf _{z \in F} C_{N}^{*}(z) .
$$

We apply this theorem to the pinned polymer model in Section 3. The measures $\mu$, $v \in \mathcal{K}_{d, \alpha}^{\infty}$ are interpreted physically as follows: the energy of polymer is determined by $v$. Intuitively, when a moving particle hits the fine support of the measure $v$, the energy increases. The functional $A_{t}^{v}$ is regarded as the total energy of a polymer of the length $t$. Here a moving particle is controlled by the law of the underlying process weighted by the Feynman-Kac functional $\exp \left(A_{t}^{\mu}\right)$. The simplest model is that the underlying process is the 1-dimensional Brownian motion and the measures are $\mu=v=\delta_{0}$. One can compute a spectral function exactly and show the differentiability of it (see for example, [20, Example 3.1]). For each $\beta \geq 0$, the polymer is pinned and the upper bound of $\kappa$ satisfying (1.3) is described by $\beta$. In [2], the case where $\mu$ equals $v$ is treated. However employing Theorem 1.1, we need not assume that $\mu$ equals $v$. Theorem 3.3 says that the polymer model has a critical inverse 
temperature $\beta_{\mathrm{cr}}>0$ such that the polymer is pinned for every $\beta>\beta_{\mathrm{cr}}$, but is not for $\beta \leq \beta_{\mathrm{cr}}$. That is, the polymer has a phase transition for pinning. We identify the critical value $\beta_{\mathrm{cr}}$ in (3.2). The critical value $\beta_{\mathrm{cr}}$ is strictly positive if the underlying process is transient. However $\beta_{\mathrm{cr}}$ is equal to zero if it is recurrent. Moreover, for $\beta>\beta_{\mathrm{cr}}$, the supremum of $\kappa$ satisfying (1.3) is equal to

$$
z_{0}(\beta)=\left.\frac{d}{d \lambda} C^{(\beta)}(\lambda)\right|_{\lambda=0} .
$$

In [6], this result is obtained for Brownian motions, and our result is regarded as a natural extension to symmetric $\alpha$-stable processes. At the last of Section 3, we compute $z_{0}(\beta)$ explicitly for the 1-dimensional symmetric $\alpha$-stable process with $1<\alpha<2$ and $\mu=v=\delta_{0}$.

The sections from Section 4 are devoted to the proof of Theorem 1.1. The Gärtner-Ellis theorem is well-known as a useful method for the proof of the LDP. To apply the Gärtner-Ellis theorem, we need to show the following two facts, namely, the existence of the logarithmic moment generating function $C_{N}(\lambda)$ and the differentiability of it. We define a function $C(\lambda)$ by

$$
C(\lambda)=\lim _{t \rightarrow \infty} \frac{1}{t} \log E_{x}\left[\exp \left(A_{t}^{\mu}+\lambda A_{t}^{v}\right)\right], \quad \lambda \in \boldsymbol{R} .
$$

The limit (1.6) coincides with the bottom of the spectrum of the Schrödinger type operator

$$
\mathcal{H}^{\mu+\lambda \nu}=\frac{1}{2}(-\Delta)^{\alpha / 2}-\mu-\lambda \nu, \quad \lambda \in \boldsymbol{R} .
$$

Namely,

$$
(1.8) C(\lambda)=-\inf \left\{\mathcal{E}^{(\alpha)}(u, u)-\int_{\boldsymbol{R}^{d}} u^{2} d \mu-\lambda \int_{\boldsymbol{R}^{d}} u^{2} d v ; u \in \mathcal{D}\left(\mathcal{E}^{(\alpha)}\right), \int_{\boldsymbol{R}^{d}} u^{2} d x=1\right\}
$$

where $\left(\mathcal{E}^{(\alpha)}, \mathcal{D}\left(\mathcal{E}^{(\alpha)}\right)\right), 0<\alpha<2$, is the Dirichlet form generated by the symmetric $\alpha$ stable process. The function $C(\lambda)$ is called a spectral function. The existence of the limit (1.6) (and thus (1.4)) follows from the $L^{p}$-independence of growth bound, which is shown for Brownian motions in Simon [18] and for more general symmetric Markov processes in Takeda [21]. Several results on the differentiability of $C_{N}(\lambda)$ are known for $\mu \equiv 0$. When $\alpha=2$ and the potential $v$ is a function in a certain Kato class, Arendt and Batty [3] prove that the function $C(\lambda)$ is differentiable at $\lambda=0$. More generally, in the case that $0<\alpha \leq 2$ and the potential $v$ is a signed measure in the Green-tight Kato class, Takeda and Tsuchida [23] show the differentiability. We here prove the differentiability by the argument similar to [23]. Set

$$
\lambda_{0}=\sup \{\lambda \in \boldsymbol{R} ; C(\lambda)=0\} .
$$

For $\lambda>\lambda_{0},-C(\lambda)$ is the principal eigenvalue of the operator $\mathcal{H}^{\mu+\lambda v}$, thus $C(\lambda)$ is differentiable on $\left(\lambda_{0}, \infty\right)$ by the analytic perturbation theory in [11, Chapter VII]. For $\lambda<\lambda_{0}$, we get $C(\lambda)=0$ by the same argument as in [22, Lemma 5.1]. Thus it is enough to show the differentiability of $C(\lambda)$ at $\lambda=\lambda_{0}$. We see from Theorem 1.2 that $C(\lambda)$ is differentiable at $\lambda_{0}$, if $\mathcal{H}^{\mu+\lambda_{0} v}$ is null critical, that is, any $\mathcal{H}^{\mu+\lambda_{0} v}$-harmonic function $h$ (i.e., $\mathcal{H}^{\mu+\lambda_{0} v} h=0$ ) is 
not square integrable. We show in Theorem 4.25 the null criticality of it by using the Harnack inequality.

For a signed measure $v$ in $\mathcal{K}_{d, \alpha}^{\infty}$, Takeda and Tsuchida constructed the $\mathcal{H}^{\lambda_{0} v}$-harmonic function $h$ (cf. [23, Definition 4.1]) which is not in $L^{2}(d x)$ but in the extended Dirichlet space $\mathcal{D}_{e}\left(\mathcal{E}^{(\alpha)}\right)$. They first obtain a function $u_{0}$ attaining the infimum of $-C\left(\lambda_{0}\right)$ by applying the compactness of the embedding of $\mathcal{D}_{e}\left(\mathcal{E}^{(\alpha)}\right)$ into $L^{2}\left(v^{+}\right)$. The $\mathcal{H}^{\lambda_{0} v}$-harmonic function $h$ is constructed as a continuous version $u_{0}$ in [23, Proposition 4.14]. If $\lambda_{0} \geq 0$, we can construct the harmonic function by the argument similar to [23]. However, we can not directly follow the argument in [23], because for $\mu \not \equiv 0$, the standing point $\lambda_{0}$ may be strictly negative due to $\int_{\boldsymbol{R}^{d}} u^{2} d \mu$ in (1.8). In Section 4, we construct the function $u_{0}$ as a minimizer of the form (4.2) or (4.3) in Lemma 4.2 and show that the function $h$ constructed by $u_{0}$ is continuous. We see from Lemma 4.2 and Proposition 4.21 that $\mathcal{H}^{\mu+\lambda_{0} v}$ is critical. We would like to make a comment on the condition $\alpha<d \leq 2 \alpha$ (of Theorem 4.25). The harmonic function $h$ is equivalent to the Green function of the stable process outside of the ball $B(1)$, i.e.,

$$
\frac{c}{|x|^{d-\alpha}} \leq h(x) \leq \frac{C}{|x|^{d-\alpha}}, \quad x \in \boldsymbol{R}^{d} \backslash B(1) .
$$

Hence the condition implies that $h$ is not square integrable and $\mathcal{H}^{\mu+\lambda_{0} v}$ is null critical. As a result, the function $C(\lambda)$ is differentiable at the point $\lambda_{0}$. We now obtain the next theorem for employing Gärtner-Ellis theorem.

THEOREM 1.2. For $\alpha<d \leq 2 \alpha$ and positive measures $\mu, v \in \mathcal{K}_{d, \alpha}^{\infty}$, the spectral function $C(\lambda)$ is differentiable for all $\lambda \in \boldsymbol{R}$.

We can show the differentiability for $d=1<\alpha$ similarly in [20].

Throughout this paper, $m$ is the Lebesgue measure, $B(R)$ an open ball with radius $R$ centered at the origin. $c, C, \ldots$ are positive constants which may be different at different occurrences. We denote $\tau_{R}$ the first exit time of the ball $B(R)$, i.e.,

$$
\tau_{R}=\inf \left\{t>0 ; X_{t} \notin B(R)\right\},
$$

and for a measure $v, d v_{R}=\mathbf{1}_{B(R)} d \nu$.

Acknowledgments. The author would like to express his gratitude to Professors Masayoshi Takeda and Kaneharu Tsuchida for their careful reading, valuable comments and numerous suggestions. He would like to thank Professor Yuichi Shiozawa for his referring to a calculation of spectral functions. His appreciation goes to the unknown referee for careful reading this paper.

2. Preliminaries. Let $\boldsymbol{M}^{\alpha}=\left(\Omega, \mathcal{F}, \mathcal{F}_{t}, \theta_{t}, P_{x}, X_{t}\right)$ be a symmetric $\alpha$-stable process on $\boldsymbol{R}^{d}$ with $0<\alpha \leq 2$. Here $\left\{\mathcal{F}_{t}\right\}_{t \geq 0}$ is the minimal (augmented) admissible filtration and $\theta_{t}, t \geq 0$, is the shift operator satisfying $X_{s}\left(\theta_{t}\right)=X_{s+t}$ identically for $s, t \geq 0$. We assume that $\alpha<d \leq 2 \alpha$, that is, the process $\boldsymbol{M}^{\alpha}$ is transient. Let $p(t, x, y)$ be the transition density function of $\boldsymbol{M}^{\alpha}$, and $P_{t}$ is the semigroup of $\boldsymbol{M}^{\alpha}$ defined by

$$
P_{t} f(x)=\int_{\boldsymbol{R}^{d}} p(t, x, y) f(y) d y=E_{x}\left[f\left(X_{t}\right)\right] .
$$


Let $G(x, y)$ be its Green function defined by

$$
G(x, y)=\int_{0}^{\infty} p(t, x, y) d t=C(d, \alpha)|x-y|^{\alpha-d},
$$

where $C(d, \alpha)=2^{1-\alpha} \pi^{-d / 2} \Gamma((d-\alpha) / 2) \Gamma(\alpha / 2)^{-1}$.

Definition 2.1. (i) A positive Radon measure $\mu$ on $\boldsymbol{R}^{d}$ is said to be in the Kato class, $\mu \in \mathcal{K}_{d, \alpha}$ in notation, if

$$
\lim _{r \rightarrow 0} \sup _{x \in \boldsymbol{R}^{d}} \int_{|x-y| \leq r} G(x, y) \mu(d y)=0 .
$$

(ii) A measure $\mu$ on $\boldsymbol{R}^{d}$ is in Green-tight Kato class, $\mu \in \mathcal{K}_{d, \alpha}^{\infty}$ in notation, if $\mu$ is in $\mathcal{K}_{d, \alpha}$ and satisfies

$$
\lim _{r \rightarrow \infty} \sup _{x \in \boldsymbol{R}^{d}} \int_{|y|>r} G(x, y) \mu(d y)=0 .
$$

Let $G_{\beta}(x, y)$ be the $\beta$-resolvent kernel of $\boldsymbol{M}^{\alpha}$, i.e.,

$$
G_{\beta}(x, y)=\int_{0}^{\infty} e^{-\beta t} p(t, x, y) d t,
$$

and $G_{\beta} \mu(x)$ the $\beta$-potential of $\mu$, i.e.,

$$
G_{\beta} \mu(x)=\int_{\boldsymbol{R}^{d}} G_{\beta}(x, y) \mu(d y) .
$$

In particular, we write the 0 -potential of $\mu$ by $G \mu(x)$ simply.

Let $\left(\mathcal{E}^{(\alpha)}, \mathcal{D}\left(\mathcal{E}^{(\alpha)}\right)\right)$ be the Dirichlet form generated by $\boldsymbol{M}^{\alpha}$, i.e., for $0<\alpha<2$,

$$
\begin{aligned}
& \mathcal{E}^{(\alpha)}(u, v)=\frac{1}{2} \mathcal{A}(d, \alpha) \iint_{\boldsymbol{R}^{d} \times \boldsymbol{R}^{d} \backslash \Delta} \frac{(u(x)-u(y))(v(x)-v(y))}{|x-y|^{d+\alpha}} d x d y, \\
& \mathcal{D}\left(\mathcal{E}^{(\alpha)}\right)=\left\{u \in L^{2}\left(\boldsymbol{R}^{d}\right) ; \iint_{\boldsymbol{R}^{d} \times \boldsymbol{R}^{d} \backslash \Delta} \frac{(u(x)-u(y))^{2}}{|x-y|^{d+\alpha}} d x d y<\infty\right\},
\end{aligned}
$$

where $\Delta=\left\{(x, x) ; x \in \boldsymbol{R}^{d}\right\}$ and

$$
\mathcal{A}(d, \alpha)=\frac{\alpha 2^{d-1} \Gamma((\alpha+d) / 2)}{\pi^{d / 2} \Gamma(1-\alpha / 2)} .
$$

For $\alpha=2, \boldsymbol{M}^{2}$ is the Brownian motion. Its Dirichlet form is the classical Dirichlet integral, that is,

$$
\mathcal{E}^{(2)}(u, v)=\frac{1}{2} \int_{\boldsymbol{R}^{d}} \nabla u \cdot \nabla v d x,
$$

and the domain $\mathcal{D}\left(\mathcal{E}^{(2)}\right)$ is the Sobolev space $H^{1}\left(\boldsymbol{R}^{d}\right)$. Let $\mathcal{D}_{e}\left(\mathcal{E}^{(\alpha)}\right)$ be the extended Dirichlet space, namely, the family of measurable functions $u$ on $\boldsymbol{R}^{d}$ such that $|u|<\infty m$-a.e. and there exists an $\mathcal{E}^{(\alpha)}$-Cauchy sequence $\left\{u_{n}\right\}$ of functions in $\mathcal{D}\left(\mathcal{E}^{(\alpha)}\right)$ such that $\lim _{n \rightarrow \infty} u_{n}=u m$-a.e. [10, p. 35]. Then $\mathcal{D}_{e}\left(\mathcal{E}^{(\alpha)}\right)$ is a Hilbert space with inner product $\mathcal{E}^{(\alpha)}$ because $\boldsymbol{M}^{\alpha}$ is transient [10, Theorem 1.5.3]. 
For $\mu \in \mathcal{K}_{d, \alpha}$, we define a symmetric bilinear form $\mathcal{E}^{\mu}$ by

$$
\mathcal{E}^{\mu}(u, u)=\mathcal{E}^{(\alpha)}(u, u)-\int_{\boldsymbol{R}^{d}} \tilde{u}^{2} d \mu, u \in \mathcal{D}\left(\mathcal{E}^{(\alpha)}\right),
$$

where $\tilde{u}$ is a quasi-continuous version of $u$ [10, Theorem 2.1.3]. In the sequel, we always assume that every function $u \in \mathcal{D}_{e}\left(\mathcal{E}^{(\alpha)}\right)$ is represented by its quasi-continuous version. Since $\mu$ charges no set of zero capacity by [1, Theorem 3.3], the form $\mathcal{E}^{\mu}$ is well defined. We see from [1, Theorem 4.1] that $\left(\mathcal{E}^{\mu}, \mathcal{D}\left(\mathcal{E}^{(\alpha)}\right)\right)$ becomes a lower semi-bounded closed symmetric form. We call $\left(\mathcal{E}^{\mu}, \mathcal{D}\left(\mathcal{E}^{(\alpha)}\right)\right)$ a Schrödinger form. Denote by $\mathcal{H}^{\mu}$ the self-adjoint operator generated by $\left(\mathcal{E}^{\mu}, \mathcal{D}\left(\mathcal{E}^{(\alpha)}\right)\right)$ where $\mathcal{E}^{\mu}(u, v)=\left(\mathcal{H}^{\mu} u, v\right)$. Let $P_{t}^{\mu}$ be the $L^{2}$-semigroup, i.e., $P_{t}^{\mu}=\exp \left(-t \mathcal{H}^{\mu}\right)$. We see from [1, Theorem 6.3(iv)] that $P_{t}^{\mu}$ admits a symmetric integral kernel $p^{\mu}(t, x, y)$ which is a jointly continuous function on $(0, \infty) \times \boldsymbol{R}^{d} \times \boldsymbol{R}^{d}$. By the Feynman-Kac formula, the semigroup $P_{t}^{\mu}$ is written as

$$
P_{t}^{\mu} f(x)=E_{x}\left[\exp \left(A_{t}^{\mu}\right) f\left(X_{t}\right)\right] .
$$

We provide known facts on the Kato class measures. The following theorem is a Poincaré type inequality which is derived by Stollman-Voigt [19].

THEOREM 2.2. Let $\mu \in \mathcal{K}_{d, \alpha}$. Then for $\beta \geq 0$,

$$
\int_{\boldsymbol{R}^{d}} u^{2} d \mu \leq\left\|G_{\beta} \mu\right\|_{\infty} \mathcal{E}_{\beta}^{(\alpha)}(u, u), u \in \mathcal{D}\left(\mathcal{E}^{(\alpha)}\right),
$$

where $\mathcal{E}_{\beta}^{(\alpha)}(u, u)=\mathcal{E}^{(\alpha)}(u, u)+\beta \int_{\boldsymbol{R}^{d}} u^{2} d x$.

We see from [12] that for $\mu \in \mathcal{K}_{d, \alpha}$,

$$
\lim _{\beta \rightarrow \infty}\left\|G_{\beta} \mu\right\|_{\infty}=0 .
$$

Therefore the equation (2.5) tells us that for any $\varepsilon>0$ there exists a positive constant $M(\varepsilon)$ such that

$$
\int_{\boldsymbol{R}^{d}} u^{2} d \mu \leq \varepsilon \mathcal{E}^{(\alpha)}(u, u)+M(\varepsilon) \int_{\boldsymbol{R}^{d}} u^{2} d x, u \in \mathcal{D}\left(\mathcal{E}^{(\alpha)}\right) .
$$

A positive function $h$ is said to be $\gamma$-excessive if $h$ satisfies $e^{-\gamma t} P_{t} h(x) \uparrow h(x)$ as $t \downarrow 0$, for any $x$. For $\gamma=0, h$ is said to be excessive simply. For $\mu \in \mathcal{K}_{d, \alpha}$, let $A_{t}^{\mu}$ be a positive continuous additive functional under the Revuz correspondence to $\mu$, i.e., for any $f \in \mathcal{B}^{+}$ and any $\gamma$-excessive function $h$ (cf. [10, p. 188]),

$$
\langle h \mu, f\rangle=\lim _{t \rightarrow 0} \frac{1}{t} E_{h m}\left[\int_{0}^{t} f\left(X_{s}\right) d A_{s}^{\mu}\right] .
$$

A measure $\mu \in \mathcal{K}_{d, \alpha}^{\infty}$ is known to be Green bounded (cf. [5, Proposition 2.2]), namely,

$$
\sup _{x \in \boldsymbol{R}^{d}} G \mu(x)=\sup _{x \in \boldsymbol{R}^{d}} E_{x}\left[A_{\infty}^{\mu}\right]<\infty .
$$

Taking $\beta=0$ in (2.5), we know that for $\mu \in \mathcal{K}_{d, \alpha}^{\infty}$

$$
\int_{\boldsymbol{R}^{d}} u^{2} d \mu \leq\|G \mu\|_{\infty} \mathcal{E}^{(\alpha)}(u, u), u \in \mathcal{D}_{e}\left(\mathcal{E}^{(\alpha)}\right),
$$


and thus $L^{2}(\mu)$ contains $\mathcal{D}_{e}\left(\mathcal{E}^{(\alpha)}\right)$.

3. Critical value $\beta_{\mathrm{cr}}$. In this section, we assume the differentiability of $C(\lambda)$ to apply Gärtner-Ellis theorem. For a sufficient condition for the differentiability of $C(\lambda)$, see Section 4 to the last.

We consider a polymer model stated in Introduction. Originally, if a polymer satisfies (1.3), it is called the pinned polymer. From the equation (1.3), we intuitively see that the polymer is pressed on the origin in the sense that the occupation time at the origin has at least liner growth. We want to extend to the polymer models defined by symmetric $\alpha$-stable processes $X_{t}$ on $\boldsymbol{R}^{d}, d \geq 2$. However, the energy of polymer, $\int_{0}^{t} \delta_{0}\left(X_{s}\right) d s$, is not welldefined for $d \geq 2$. Then we regard it as $\int_{0}^{t} \mathbf{1}_{B}\left(X_{S}\right) d s$ for a small ball $B$ centered at the origin. Moreover, we extend $\int_{0}^{t} \mathbf{1}_{B}\left(X_{s}\right) d s$ to PCAF $A_{t}^{v}$ under the Revuz correspondence to $v \in \mathcal{K}_{\alpha, d}^{\infty}$, because $\int_{0}^{t} \mathbf{1}_{B}\left(X_{s}\right) d s$ is the typical one of $\mathbf{1}_{B}(x) d x \in \mathcal{K}_{\alpha, d}^{\infty}$. Moreover, we extend the weight $\exp \left(\int_{0}^{t} \delta_{0}\left(B_{s}\right) d s\right)$ to $\exp \left(A_{t}^{\mu}\right)$ and set

$$
d P_{x, t}^{\mu}=\frac{1}{Z_{x, t}^{\mu}} \exp \left(A_{t}^{\mu}\right) d P_{x}
$$

where $Z_{x, t}^{\mu}$ is the normalizing factor. If there exists $\kappa>0$ such that

$$
\lim _{t \rightarrow \infty} P_{x, t}^{\mu}\left(\frac{A_{t}^{v}}{t}>\kappa\right)=1,
$$

then the polymer is said to be pinned. We then realize that the polymer is pressed on the fine support of $v$. By Theorem 1.1, we have

$$
\lim _{t \rightarrow \infty} \frac{1}{t} \log P_{x, t}^{\mu}\left(\frac{A_{t}^{v}}{t}>\kappa\right)=-\inf _{z>\kappa} C_{N}^{*}(z) .
$$

We denote

$$
\mathcal{N}=\left\{z \in(0, \infty) ; C_{N}^{*}(z)=0\right\} .
$$

The polymer is pinned if and only if $\mathcal{N} \neq \emptyset$. More precisely,

LEMMA 3.1. It holds that

$$
\begin{aligned}
& \lambda_{0}<0 \Longleftrightarrow \mathcal{N}=\left\{z_{0}\right\}, \\
& \lambda_{0} \geq 0 \Longleftrightarrow \mathcal{N}=\emptyset .
\end{aligned}
$$

Here

$$
z_{0}=C_{N}^{\prime}(0)=C^{\prime}(0) .
$$

Proof. Note that $C_{N}(\lambda)=C(\lambda)-C(0)$ and the spectral function $C(\lambda)$ is differentiable, thus $z_{0}$ is well-defined. Let $F(\lambda)=z_{0} \lambda+C(0)$. First we discuss the case of $\lambda_{0}<0$. The spectral function $C(\lambda)$ is strictly increasing on $\left(\lambda_{0}, \infty\right)$, which implies that $z_{0}>0$ and $C(0)>0$. We show that $z_{0}$ belongs to $\mathcal{N}$. Because $C(\lambda)$ is convex, $C(\lambda)-F(\lambda) \geq 0$. Thus

$$
C(\lambda)-\lambda z_{0}=C(\lambda)-F(\lambda)+C(0) \geq C(0) .
$$


Hence we have

On the other hand,

$$
C(0) \leq \inf _{\lambda \in \boldsymbol{R}}\left(C(\lambda)-\lambda z_{0}\right)=-C^{*}\left(z_{0}\right) .
$$

$$
C(0)=\left.\left(C(\lambda)-\lambda z_{0}\right)\right|_{\lambda=0} \geq \inf _{\lambda \in \boldsymbol{R}}\left(C(\lambda)-\lambda z_{0}\right)=-C^{*}\left(z_{0}\right) .
$$

Hence $C(0)=-C^{*}\left(z_{0}\right)$ and so $C_{N}^{*}\left(z_{0}\right)=0$ where $C_{N}^{*}(z)=C^{*}(z)+C(0)$. As a result, for each $z^{\prime}>z_{0}$, there exists $\lambda^{\prime}$ such that $C\left(\lambda^{\prime}\right)<z^{\prime} \lambda^{\prime}+C(0)$ and $0<C^{*}\left(z^{\prime}\right)+C(0)=C_{N}^{*}\left(z^{\prime}\right)$. Thus $\left(z_{0}, \infty\right) \cap \mathcal{N}=\emptyset$. By the same argument as above, for each $z^{\prime} \in\left(0, z_{0}\right)$, there exists $\lambda^{\prime}<0$ such that $z^{\prime} \lambda^{\prime}+C(0)>C\left(\lambda^{\prime}\right)$. Hence we have $C_{N}^{*}\left(z^{\prime}\right)>0$ for any $z^{\prime} \in\left(0, z_{0}\right)$, and thus $\left(0, z_{0}\right) \cap \mathcal{N}=\emptyset$. Consequently, $\mathcal{N}=\left\{z_{0}\right\}$.

If $\lambda_{0}>0$, then $C(0)=0$. Hence, for any $z>0$

$$
C_{N}^{*}(z)=C^{*}(z) \geq\left.(\lambda z-C(\lambda))\right|_{\lambda=\lambda_{0}}=\lambda_{0} z>0 .
$$

This implies that $\mathcal{N}=\emptyset$. If $\lambda_{0}=0$, the tangent of $C(\lambda)$ at $\lambda_{0}$ is $F(\lambda) \equiv 0$. For every $z>0$, there exists $\lambda^{\prime}$ such that $z \lambda^{\prime}>C\left(\lambda^{\prime}\right)$. Hence

$$
0<\lambda^{\prime} z-C\left(\lambda^{\prime}\right) \leq C_{N}^{*}(z)
$$

which implies that $\mathcal{N}=\emptyset$.

The converse is trivial.

We write $P_{x, t}^{(\beta)}=P_{x, t}^{\beta \mu}$, for $\beta \geq 0$. Let $C^{(\beta)}(\lambda)$ the spectral function corresponding to the Schrödinger type operator $\mathcal{H}^{\beta \mu+\lambda \nu}=2^{-1}(-\Delta)^{\alpha / 2}-\beta \mu-\lambda \nu$. Theorem 1.1 tells us that

$$
\lim _{t \rightarrow \infty} \frac{1}{t} \log P_{x, t}^{(\beta)}\left(\frac{A_{t}^{v}}{t}>\kappa\right)=-\inf _{z>\kappa} C_{N}^{(\beta) *}(z),
$$

where $C_{N}^{(\beta) *}(z)$ is the Fenchel-Legendre transform of $C_{N}^{(\beta)}(\lambda)$. We set, for $\beta>0$,

$$
\lambda_{0}(\beta)=\sup \left\{\lambda \in \boldsymbol{R} ; C^{(\beta)}(\lambda)=0\right\},
$$

and

$$
\beta_{\mathrm{cr}}=\sup \left\{\beta \in \boldsymbol{R} ; C^{(\beta)}(0)=0\right\} .
$$

We see from [20, Lemma 4.2] that if the underlying process is recurrent, then $\beta_{\mathrm{cr}}=0$, on the other hand if it is transient, then $\beta_{\mathrm{cr}}>0$. Hence we see that for $\alpha<d \leq 2 \alpha, \beta_{\mathrm{cr}}$ is strictly positive.

LEMMA 3.2. It holds that

$$
\begin{aligned}
0 \leq \beta \leq \beta_{\mathrm{cr}} & \Longleftrightarrow \lambda_{0}(\beta) \geq 0, \\
\beta>\beta_{\mathrm{cr}} & \Longleftrightarrow \lambda_{0}(\beta)<0 .
\end{aligned}
$$

Proof. We consider $C^{(\beta)}(0)$ as a function of $\beta$. Then it is strictly increasing [23, Lemma 2.4]. Hence, for $\beta>\beta_{\mathrm{cr}}$,

$$
C^{(\beta)}(0)=-\inf \left\{\mathcal{E}^{(\alpha)}(u, u)-\beta \int_{\boldsymbol{R}^{d}} u^{2} d \mu ; \int_{\boldsymbol{R}^{d}} u^{2} d x=1\right\}>0 .
$$


In addition, for fixed $\beta, C^{(\beta)}(\lambda)$ is strictly increasing in $\lambda$ on $\left(\lambda_{0}(\beta), \infty\right)$ and $C^{(\beta)}(\lambda)$ is equal to zero on $\left(-\infty, \lambda_{0}(\beta)\right)$ by the definition of $\lambda_{0}(\beta)$.

For $0 \leq \beta \leq \beta_{\mathrm{cr}}, C^{(\beta)}(0)$ is equal to zero, This implies $\lambda_{0}(\beta) \geq 0$. For $\beta>\beta_{\mathrm{cr}}$, $C^{(\beta)}(0)>0$, and $\lambda_{0}(\beta)$ must be negative.

Denote

$$
z_{0}(\beta)=\left.\frac{d}{d \lambda} C^{(\beta)}(\lambda)\right|_{\lambda=0} .
$$

By Lemma 3.1 and Lemma 3.2, we have next theorem, which is an extension of Cranston and Molchanov [7, Corollary 4.2] to symmetric $\alpha$-stable processes; they consider a polymer model made of simple random walks whose energy is defined by the local time at the origin.

THEOREM 3.3. If $\beta>\beta_{\mathrm{cr}}$, then for any $0<\kappa<z_{0}(\beta)$,

$$
\lim _{t \rightarrow \infty} P_{x, t}^{(\beta)}\left(\frac{A_{t}^{v}}{t}>\kappa\right)=1 .
$$

If $0 \leq \beta \leq \beta_{\mathrm{cr}}$, then for any $\kappa>0$, the limit of (3.3) is less than 1 , as $t \rightarrow \infty$.

For $\beta \geq 0, d=1,1<\alpha \leq 2$ and $\mu=v=\delta_{0}$, we can compute the spectral function $C(\lambda)$ explicitly by [17, Example 4.4],

$$
C^{(\beta)}(\lambda)= \begin{cases}C_{\alpha}(\lambda+\beta)^{\alpha /(\alpha-1)}, & \lambda+\beta \geq 0, \\ 0, & \lambda+\beta<0,\end{cases}
$$

where $C_{\alpha}=\left(\frac{2^{1 / \alpha}}{\alpha \sin (\pi / \alpha)}\right)^{\alpha /(\alpha-1)}$. Thus $C^{(\beta)}(\lambda)$ is differentiable, and so LDP holds by GärtnerEllis theorem. In addition, we have $\beta_{\mathrm{cr}}=0$ and

$$
z_{0}(\beta)=\left.\frac{d}{d \lambda} C^{(\beta)}(\lambda)\right|_{\lambda=0}=\frac{\alpha}{\alpha-1} C_{\alpha} \beta^{1 /(\alpha-1)} .
$$

Let us compute the rate function $C_{N}^{(\beta) *}(z)$. For $z>0$,

$$
\begin{aligned}
C^{(\beta) *}(z) & =\max \left\{\sup _{\lambda \geq-\beta}\left(\lambda z-C^{(\beta)}(\lambda)\right), \sup _{\lambda<-\beta}\left(\lambda z-C^{(\beta)}(\lambda)\right)\right\} \\
& =\max \left\{\sup _{\lambda \geq-\beta}\left(\lambda z-C_{\alpha}(\lambda+\beta)^{\alpha /(\alpha-1)}\right),-\beta z\right\} \\
& =\max \left\{\frac{1}{\alpha}\left(\frac{\alpha C_{\alpha}}{\alpha-1}\right)^{1-\alpha} z^{\alpha}-\beta z,-\beta z\right\}=\frac{1}{\alpha}\left(\frac{\alpha C_{\alpha}}{\alpha-1}\right)^{1-\alpha} z^{\alpha}-\beta z .
\end{aligned}
$$

It is easy for us to compute $C^{(\beta) *}(z)$ in the case of $z \leq 0$. Because $C_{N}^{(\beta) *}(z)=C^{(\beta) *}(z)+$ $C^{(\beta)}(0)$, we have

$$
C_{N}^{(\beta) *}(z)= \begin{cases}\frac{1}{\alpha}\left(\frac{\alpha C_{\alpha}}{\alpha-1}\right)^{1-\alpha} z^{\alpha}+C_{\alpha} \beta^{\alpha /(\alpha-1)}-\beta z, & z>0, \\ C_{\alpha} \beta^{\alpha /(\alpha-1)}, & z=0 \\ \infty, & z<0\end{cases}
$$


Thus if $\kappa$ is less than $z_{0}(\beta)$, then $z=z_{0}(\beta)$ attains an infimum of $C_{N}^{(\beta) *}(z)$ such that $C_{N}^{(\beta) *}\left(z_{0}(\beta)\right)=0$. Consequently, for any $\beta>0$, the polymer is pinned under $\kappa<z_{0}(\beta)$. Particularly, $\alpha=2$ means a case of Brownian motion. In this case, for any $\beta>0, z_{0}(\beta)=\beta$.

4. Construction of ground states. We use the same argument as in [23] to construct a ground state of the critical Schrödinger operator $\mathcal{H}^{\mu+\lambda_{0} \nu}$. In this section, we assume that $\lambda_{0}<0$, because the proof for $\lambda_{0} \geq 0$ is included in [23, Section 4]. Here $\lambda_{0}$ is the constant defined in (1.9).

The spectral function $C(\lambda)$ is defined by the bottom of spectrum of $\mathcal{H}^{\mu+\lambda v}$, i.e., for $\mu$, $v \in \mathcal{K}_{d, \alpha}^{\infty}$,

$$
C(\lambda)=-\inf \left\{\mathcal{E}^{\mu+\lambda v}(u, u) ; u \in \mathcal{D}\left(\mathcal{E}^{(\alpha)}\right), \int_{\boldsymbol{R}^{d}} u^{2} d x=1\right\}, \lambda \in \boldsymbol{R} .
$$

It is known in [21] that the function $C(\lambda)$ coincides with

$$
C(\lambda)=\lim _{t \rightarrow \infty} \frac{1}{t} \log E_{x}\left[\exp \left(A_{t}^{\mu}+\lambda A_{t}^{v}\right)\right] .
$$

The next lemma is a key to construct a harmonic function of $\mathcal{H}^{\mu+\lambda_{0} v}$ (see Definition 4.4 below).

LeMma 4.1 ([23, Lemma 3.1]). If $\mu \in \mathcal{K}_{d, \alpha}^{\infty}$, then the embedding of $\mathcal{D}_{e}\left(\mathcal{E}^{(\alpha)}\right)$ into $L^{2}(\mu)$ is compact.

LEMMA 4.2. For $v, \mu \in \mathcal{K}_{d, \alpha}^{\infty}$, the number $\lambda_{0}<0$ is characterized as follows;

$$
\inf \left\{\mathcal{E}^{(\alpha)}(u, u)-\lambda_{0} \int_{\boldsymbol{R}^{d}} u^{2} d v ; u \in \mathcal{D}_{e}\left(\mathcal{E}^{(\alpha)}\right), \int_{\boldsymbol{R}^{d}} u^{2} d \mu=1\right\}=1 .
$$

Lemma 4.1 tells us that a minimizing sequence in (4.2) or (4.3) below exists.

Proof. Let us denote

$$
F(\lambda)=\inf \left\{\mathcal{E}^{(\alpha)}(u, u)-\lambda \int_{\boldsymbol{R}^{d}} u^{2} d \nu ; u \in \mathcal{D}_{e}\left(\mathcal{E}^{(\alpha)}\right), \int_{\boldsymbol{R}^{d}} u^{2} d \mu=1\right\} .
$$

First we show $F\left(\lambda_{0}\right)=1$ by the same argument as in [23, Lemma 2.2]. Suppose $F\left(\lambda_{0}\right)<1$. Then we can choose $\psi_{0} \in C_{0}^{\infty}\left(\boldsymbol{R}^{d}\right)$ with $\int_{\boldsymbol{R}^{d}} \psi_{0}^{2} d \mu=1$ such that

$$
\mathcal{E}^{(\alpha)}\left(\psi_{0}, \psi_{0}\right)-\lambda_{0} \int_{\boldsymbol{R}^{d}} \psi_{0}^{2} d v<1
$$

Hence it holds that

$$
\mathcal{E}^{(\alpha)}\left(\psi_{0}, \psi_{0}\right)-\int_{\boldsymbol{R}^{d}} \psi_{0}^{2} d \mu-\lambda_{0} \int_{\boldsymbol{R}^{d}} \psi_{0}^{2} d \nu<0 .
$$

Let

$$
u_{0}=\frac{\psi_{0}}{\sqrt{\int_{\boldsymbol{R}^{d}} \psi_{0}^{2} d x}} .
$$


Then $\int_{\boldsymbol{R}^{d}} u_{0}^{2} d x=1$ and

$$
\mathcal{E}^{(\alpha)}\left(u_{0}, u_{0}\right)-\int_{\boldsymbol{R}^{d}} u_{0}^{2} d \mu-\lambda_{0} \int_{\boldsymbol{R}^{d}} u_{0}^{2} d v<0 .
$$

This contradicts $C\left(\lambda_{0}\right)=0$.

Next we suppose $F\left(\lambda_{0}\right)>1$. Then there exists $\lambda^{+} \in\left(\lambda_{0}, 0\right)$ such that $F\left(\lambda^{+}\right)>1$ because of the continuity of the function $F(\lambda)$. From $\lambda^{+}>\lambda_{0}$, we see that $C\left(\lambda^{+}\right)>0$. Hence we can choose $\psi_{0} \in C_{0}^{\infty}\left(\boldsymbol{R}^{d}\right)$ such that

$$
\mathcal{E}^{(\alpha)}\left(\psi_{0}, \psi_{0}\right)-\int_{\boldsymbol{R}^{d}} \psi_{0}^{2} d \mu-\lambda^{+} \int_{\boldsymbol{R}^{d}} \psi_{0}^{2} d v<0 .
$$

Letting

$$
u_{0}=\frac{\psi_{0}}{\sqrt{\int_{\boldsymbol{R}^{d}} \psi_{0}^{2} d \mu}}
$$

we have $\int_{\boldsymbol{R}^{d}} u_{0}^{2} d \mu=1$ and

$$
\mathcal{E}^{(\alpha)}\left(u_{0}, u_{0}\right)-\lambda^{+} \int_{\boldsymbol{R}^{d}} u_{0}^{2} d v<1 .
$$

This implies that $F\left(\lambda^{+}\right)<1$, which is contrary to $F\left(\lambda^{+}\right)>1$. Consequently, we have $F\left(\lambda_{0}\right)=1$.

Finally we show the uniqueness of $\lambda_{0}$ satisfying (4.2). For any $\lambda>\lambda_{0}, C(\lambda)>0$ and so $F(\lambda)<1$. We suppose that $F\left(\lambda_{1}\right)=1$ for some $\lambda_{1}<\lambda_{0}$. Since the embedding of $\mathcal{D}_{e}\left(\mathcal{E}^{(\alpha)}\right)$ to $L^{2}(\mu)$ is compact by Lemma 4.1, there exists the function $u_{1}$ in $\mathcal{D}_{e}\left(\mathcal{E}^{(\alpha)}\right)$ such that $u_{1}$ attains the infimum of $F\left(\lambda_{1}\right)(=1)$. Because of the irreducibility of $\boldsymbol{M}^{\alpha}$, the function $u_{1}$ is strictly positive $v$-a.e. Therefore

$$
\mathcal{E}^{(\alpha)}\left(u_{1}, u_{1}\right)-\lambda_{0} \int_{\boldsymbol{R}^{d}} u_{1}^{2} d v<\mathcal{E}^{(\alpha)}\left(u_{1}, u_{1}\right)-\lambda_{1} \int_{\boldsymbol{R}^{d}} u_{1}^{2} d v=1,
$$

which contradicts $F\left(\lambda_{0}\right)=1$.

REMARK 4.3. We see from [23, Lemma 2.4.] that if $\lambda_{0} \geq 0$, then

$$
\inf \left\{\mathcal{E}^{(\alpha)}(u, u) ; u \in \mathcal{D}_{e}\left(\mathcal{E}^{(\alpha)}\right), \int_{\boldsymbol{R}^{d}} u^{2} d \mu+\lambda_{0} \int_{\boldsymbol{R}^{d}} u^{2} d v=1\right\}=1 .
$$

This can be proved by the same argument as in the proof of Lemma 4.2.

In general, for a signed measure $\mu=\mu^{+}-\mu^{-} \in \mathcal{K}_{d, \alpha}^{\infty}-\mathcal{K}_{d, \alpha}^{\infty}$, the operator $\mathcal{H}^{\mu}=$ $1 / 2(-\Delta)^{\alpha / 2}-\mu^{+}+\mu^{-}$is said to be subcritical, if $\mathcal{H}^{\mu}$ possesses the minimal positive Green function $G^{\mu}$, that is,

$$
G^{\mu}(x, y)=\int_{0}^{\infty} p^{\mu}(t, x, y) d t<\infty, \quad x \neq y .
$$


It was shown in [24] that a necessary and sufficient condition for the operator $\mathcal{H}^{\mu}$ being subcritical is

$$
\inf \left\{\mathcal{E}^{(\alpha)}(u, u)+\int_{\boldsymbol{R}^{d}} u^{2} d \mu^{-} ; u \in \mathcal{D}_{e}\left(\mathcal{E}^{(\alpha)}\right), \int_{\boldsymbol{R}^{d}} u^{2} d \mu^{+}=1\right\}>1 .
$$

Lemma 4.2 tells us that $\mathcal{H}^{\mu+\lambda_{0} v}$ is not subcritical.

We can choose the function which attains the infimum of (4.2) as follows. There is a minimizing sequence $\left\{u_{n}\right\} \subset \mathcal{D}_{e}\left(\mathcal{E}^{(\alpha)}\right)$ of (4.2) such that

$$
\lim _{n \rightarrow \infty}\left(\mathcal{E}^{(\alpha)}\left(u_{n}, u_{n}\right)-\lambda_{0} \int_{\boldsymbol{R}^{d}} u_{n}^{2} d \nu\right)=1 \text { with } \int_{\boldsymbol{R}^{d}} u_{n}^{2} d \mu=1 \text { for each } n .
$$

Let us denote $\mathcal{E}^{\lambda_{0} v}(u, u)=\mathcal{E}^{(\alpha)}(u, u)-\lambda_{0} \int_{\boldsymbol{R}^{d}} u^{2} d \nu$. Because of $\lambda_{0}<0$, the symmetric form $\mathcal{E}^{\lambda_{0} v}$ is non-negative. Thus $\left(\mathcal{D}_{e}\left(\mathcal{E}^{(\alpha)}\right), \mathcal{E}^{\lambda_{0} v}\right)$ is a Hilbert space. Since the minimizing sequence $\left\{u_{n}\right\}$ is bounded in $\mathcal{E}^{\lambda_{0} v}$-norm, we have a subsequence $\left\{u_{n_{j}}\right\}$ which converges to some $u_{0} \in \mathcal{D}_{e}\left(\mathcal{E}^{(\alpha)}\right)$ weakly. Moreover, since the embedding of $\mathcal{D}_{e}\left(\mathcal{E}^{(\alpha)}\right)$ to $L^{2}(\mu)$ is compact, there is a subsequence $\left\{u_{n}^{\prime}\right\}$ of $\left\{u_{n_{j}}\right\}$ which strongly converges to $u_{0}$ in $L^{2}(\mu)$. Therefore $\int_{\boldsymbol{R}^{d}} u_{0}^{2} d \mu=\lim _{n \rightarrow \infty} \int_{\boldsymbol{R}^{d}} u_{n}^{\prime 2} d \mu=1$, and thus $\mathcal{E}^{\lambda_{0} \nu}\left(u_{0}, u_{0}\right) \geq 1$. On the other hand, by the uniform boundedness principle,

$$
\mathcal{E}^{\lambda_{0} v}\left(u_{0}, u_{0}\right) \leq \liminf _{n \rightarrow \infty} \mathcal{E}^{\lambda_{0} v}\left(u_{n}^{\prime}, u_{n}^{\prime}\right)=1 .
$$

Thus $u_{0}$ attains the infimum of (4.2). We show that the function $u_{0}$ has a continuous version.

We define the $\mathcal{H}^{\mu}$-harmonicity probabilistically.

DEFINITION 4.4 ([23, Definition 4.1]). A bounded finely continuous function $h$ on $\boldsymbol{R}^{d}$ is said to be $\mathcal{H}^{\mu}$-harmonic, if for any relatively compact domain $D \subset \boldsymbol{R}^{d}$,

$$
h(x)=E_{x}\left[\exp \left(A_{\tau_{D}}^{\mu}\right) h\left(X_{\tau_{D}}\right)\right], \quad x \in D .
$$

Here $\tau_{D}$ is the first exit time from $D$, i.e., $\tau_{D}=\inf \left\{t>0 ; X_{t} \notin D\right\}$.

A operator $\mathcal{H}$ is said to be critical, if $\mathcal{H}$ is not subcritical and admits a positive $\mathcal{H}$ harmonic function [15, p. 145]. Remark that the operator $\mathcal{H}^{\mu+\lambda_{0} v}$ is not subcritical (see Lemma 4.2).

For an excessive function $h$, we define $p^{h}(t, x, y)$ by

$$
p^{h}(t, x, y)=\frac{1}{h(x)} p(t, x, y) h(y), \quad t>0, x, y \in\{0<h<\infty\},
$$

and the associated semigroup $P_{t}^{h}$ by

$$
P_{t}^{h} f(x)=\int_{\{0<h<\infty\}} p^{h}(t, x, y) f(y) d y .
$$

Then $p^{h}(t, x, y)$ becomes a transition probability density because

$$
P_{t}^{h} 1(x)=\frac{1}{h(x)} P_{t} h(x) \leq \frac{h(x)}{h(x)}=1 .
$$

We call the process generated by $p^{h}$ Doob's $h$-transformed process. 
Lemma 4.5. A positive $\mathcal{H}^{\mu+v}$-harmonic function $h$ is $P_{t}^{\mu+v}$-excessive.

PROOF. Since the the semigroup $P_{t}^{\mu}$ is strongly continuous, $\lim _{t \rightarrow 0} P_{t}^{\mu+v} h(x)=h(x)$. For $m>0$, let $\tau_{m}$ be the first exit time from $B(m)$. By Definition 4.4, the function $h$ satisfies

$$
h(x)=E_{x}\left[\exp \left(A_{\tau_{n}}^{\mu+v}\right) h\left(X_{\tau_{n}}\right)\right], \quad x \in B(m)
$$

for any $n>m$. By the Markov property,

$$
\begin{aligned}
& E_{x}\left[\exp \left(A_{t}^{\mu+v}\right) h\left(X_{t}\right) ; t<\tau_{m}\right] \\
& \quad=E_{x}\left[\exp \left(A_{t}^{\mu+v}\right) E_{X_{t}}\left[\exp \left(A_{\tau_{n}}^{\mu+v}\right) h\left(X_{\tau_{n}}\right)\right] ; t<\tau_{m}\right] \\
& \quad=E_{x}\left[\exp \left(A_{t}^{\mu+v}\right) E_{x}\left[\exp \left(A_{\tau_{n}}^{\mu+v} \circ \theta_{t}\right) h\left(X_{\tau_{n}} \circ \theta_{t}\right) \mid \mathcal{F}_{t}\right] ; t<\tau_{m}\right] \\
& \quad=E_{x}\left[\exp \left(A_{t}^{\mu+v}+A_{\tau_{n}}^{\mu+v} \circ \theta_{t}\right) h\left(X_{\tau_{n}} \circ \theta_{t}\right) ; t<\tau_{m}\right] \\
& \quad=E_{x}\left[\exp \left(A_{\tau_{n}}^{\mu+v}\right) h\left(X_{\tau_{n}}\right) ; t<\tau_{m}\right] \leq h(x) .
\end{aligned}
$$

Because $\tau_{m} \rightarrow \infty$ as $m \rightarrow \infty$, and so

$$
E_{x}\left[\exp \left(A_{t}^{\mu+v}\right) h\left(X_{t}\right) ; t<\tau_{m}\right] \rightarrow E_{x}\left[\exp \left(A_{t}^{\mu+v}\right) h\left(X_{t}\right)\right]=P_{t}^{\mu+v} h(x),
$$

we have

$$
P_{t}^{\mu+v} h(x)=\lim _{m \rightarrow \infty} E_{x}\left[\exp \left(A_{t}^{\mu+v}\right) h\left(X_{t}\right) ; t<\tau_{m}\right] \leq h(x)
$$

If $\boldsymbol{M}^{\lambda_{0} v}$ is the subprocess of $\boldsymbol{M}^{\alpha}$ by the multiplicative function $\exp \left(\lambda_{0} A_{t}^{v}\right)$, then $u_{0}$ is the first eigenfunction corresponding to the generator of the time changed process of $\boldsymbol{M}^{\lambda_{0} v}$ by $A_{t}^{\mu}$. The time changed process is irreducible, because of $\int_{\boldsymbol{R}^{d}} G^{\lambda_{0} \nu}(x, y) \mu(d y)>0$. Hence $u_{0}>0, \mu$-a.e by [8, Theorem 7.3].

Let $u_{0}$ be the function attaining the infimun of (4.2).

LEMMA 4.6. The measure $u_{0} \mu$ is of finite energy integral with respect to $\mathcal{E}^{\lambda_{0} \nu}$.

Proof. By Theorem 2.2 and $\int_{\boldsymbol{R}^{d}} u_{0}^{2} d \mu=1$, for any $f \in \mathcal{D}_{e}\left(\mathcal{E}^{(\alpha)}\right)$,

$$
\begin{aligned}
\int_{\boldsymbol{R}^{d}}|f| u_{0} d \mu & \leq\left(\int_{\boldsymbol{R}^{d}} f^{2} d \mu\right)^{1 / 2}\left(\int_{\boldsymbol{R}^{d}} u_{0}^{2} d \mu\right)^{1 / 2} \\
& \leq\left(\|G \mu\|_{\infty} \mathcal{E}^{(\alpha)}(f, f)\right)^{1 / 2} \\
& \leq\left\{\|G \mu\|_{\infty}\left(\mathcal{E}^{(\alpha)}(f, f)-\lambda_{0} \int f^{2} d \nu\right)\right\}^{1 / 2} \\
& =\|G \mu\|_{\infty}^{1 / 2}\|f\|_{\mathcal{E}^{\lambda_{0} \nu}} .
\end{aligned}
$$

REMARK 4.7. If $\lambda_{0} \geq 0$, the measure $u_{0}\left(\mu+\lambda_{0} v\right)$, where $u_{0} \in \mathcal{D}_{e}\left(\mathcal{E}^{(\alpha)}\right)$ attains the infimum of (4.3), is of finite energy integral with respect to $\mathcal{E}^{(\alpha)}$. In fact, for $\eta\left(\lambda_{0}\right):=$ 
$\mu+\lambda_{0} v \in \mathcal{K}_{d, \alpha}^{\infty}$, we see that

$$
\begin{aligned}
\int_{\boldsymbol{R}^{d}}|f| u_{0} d \eta\left(\lambda_{0}\right) & \leq\left(\int_{\boldsymbol{R}^{d}} f^{2} d \eta\left(\lambda_{0}\right)\right)^{1 / 2}\left(\int_{\boldsymbol{R}^{d}} u_{0}^{2} d \eta\left(\lambda_{0}\right)\right)^{1 / 2} \\
& \leq\left(\left\|G \eta\left(\lambda_{0}\right)\right\|_{\infty} \mathcal{E}^{(\alpha)}(f, f)\right)^{1 / 2} \\
& =\left\|G \eta\left(\lambda_{0}\right)\right\|_{\infty}^{1 / 2}\|f\|_{\mathcal{E}^{(\alpha)}} .
\end{aligned}
$$

The function $u_{0}$ is also characterized as

$$
\mathcal{E}^{(\alpha)}\left(u_{0}, f\right)-\lambda_{0} \int_{\boldsymbol{R}^{d}} u_{0} f d \nu=\int_{\boldsymbol{R}^{d}} u_{0} f d \mu, \text { for all } f \in \mathcal{D}_{e}\left(\mathcal{E}^{(\alpha)}\right) .
$$

Hence Lemma 4.6 implies that for all $f \in \mathcal{D}_{e}\left(\mathcal{E}^{(\alpha)}\right)$,

$$
\mathcal{E}^{\lambda_{0} v}\left(u_{0}, f\right)=\int_{\boldsymbol{R}^{d}} u_{0} f d \mu=\mathcal{E}^{\lambda_{0} v}\left(G^{\lambda_{0} v}\left(u_{0} \mu\right), f\right) .
$$

We see, in addition, that

$$
\begin{aligned}
u_{0}(x) & =G^{\lambda_{0} v}\left(u_{0} \mu\right)(x) \\
& =E_{x}\left[\int_{0}^{\infty} \exp \left(\lambda_{0} A_{t}^{v}\right) u_{0}\left(X_{t}\right) d A_{t}^{\mu}\right] \\
& >0, \quad \text { m-a.e. }
\end{aligned}
$$

We define the function $h$ by

$$
h(x)=E_{x}\left[\int_{0}^{\infty} \exp \left(\lambda_{0} A_{t}^{v}\right) u_{0}\left(X_{t}\right) d A_{t}^{\mu}\right] .
$$

REMARK 4.8. For $\lambda_{0} \geq 0$, the function $h$ is the same one as defined by (4.8) in [23], i.e.,

$$
h(x)=E_{x}\left[\int_{0}^{\infty} u_{0}\left(X_{t}\right) d A_{t}^{\mu+\lambda_{0} v}\right] .
$$

We now prove that $h$ is a bounded continuous function.

LEMMA 4.9. The function $h$ is finely continuous.

Proof. By [10, Theorem A.2.7], we have only to show the right continuity of $h\left(X_{S}\right)$ in $s$. Because of the Markov property,

$$
\begin{aligned}
h\left(X_{S}\right) & =E_{X_{s}}\left[\int_{0}^{\infty} \exp \left(\lambda_{0} A_{t}^{v}\right) u_{0}\left(X_{t}\right) d A_{t}^{\mu}\right] \\
& =E_{x}\left[\int_{0}^{\infty} \exp \left(\lambda_{0} A_{t}^{v}\left(\theta_{s}\right)\right) u_{0}\left(X_{t+s}\right) d A_{t}^{\mu}\left(\theta_{s}\right) \mid \mathcal{F}_{s}\right] .
\end{aligned}
$$

Noting $A_{t}^{\mu}\left(\theta_{s}\right)=A_{t+s}^{\mu}-A_{s}^{\mu}$ and so $d A_{t}^{\mu}\left(\theta_{s}\right)=d A_{t+s}^{\mu}$, we see that the right-hand side equals

$$
E_{x}\left[\int_{0}^{\infty} \exp \left\{\lambda_{0}\left(A_{t+s}^{v}-A_{s}^{v}\right)\right\} u_{0}\left(X_{t+s}\right) d A_{t+s}^{\mu} \mid \mathcal{F}_{s}\right]
$$




$$
=\exp \left(-\lambda_{0} A_{s}^{v}\right) E_{x}\left[\int_{0}^{\infty} \exp \left(\lambda_{0} A_{t+s}^{v}\right) u_{0}\left(X_{t+s}\right) d A_{t+s}^{\mu} \mid \mathcal{F}_{s}\right]
$$

Setting $u=s+t$, we have

$$
\begin{aligned}
& E_{x}\left[\int_{s}^{\infty} \exp \left(\lambda_{0} A_{u}^{v}\right) u_{0}\left(X_{u}\right) d A_{u}^{\mu} \mid \mathcal{F}_{s}\right] \\
& \quad=E_{x}\left[\int_{0}^{\infty} \exp \left(\lambda_{0} A_{u}^{v}\right) u_{0}\left(X_{u}\right) d A_{u}^{\mu} \mid \mathcal{F}_{s}\right]-E_{x}\left[\int_{0}^{s} \exp \left(\lambda_{0} A_{u}^{v}\right) u_{0}\left(X_{u}\right) d A_{u}^{\mu} \mid \mathcal{F}_{s}\right],
\end{aligned}
$$

and thus

$$
\begin{aligned}
h\left(X_{s}\right)= & \exp \left(-\lambda_{0} A_{s}^{v}\right)\left\{E_{x}\left[\int_{0}^{\infty} \exp \left(\lambda_{0} A_{u}^{v}\right) u_{0}\left(X_{u}\right) d A_{u}^{\mu} \mid \mathcal{F}_{s}\right]\right. \\
& \left.-\int_{0}^{s} \exp \left(\lambda_{0} A_{u}^{v}\right) u_{0}\left(X_{u}\right) d A_{u}^{\mu}\right\} .
\end{aligned}
$$

Since the filtration $\mathcal{F}_{s}$ is right continuous, the first term is also right continuous. Consequently, we have the right continuity of $h\left(X_{s}\right)$.

We see from [10, Lemma 4.1.5] that if $h(x)=u_{0}(x) m$-a.e., then $h(x)=u_{0}(x)$ q.e. Hence [10, Theorem 4.1.2] leads us to the next lemma.

LEMMA 4.10. The function $h$ is strictly positive and satisfies

$$
h(x)=E_{x}\left[\int_{0}^{\infty} \exp \left(\lambda_{0} A_{t}^{v}\right) h\left(X_{t}\right) d A_{t}^{\mu}\right],
$$

for all $x \in \boldsymbol{R}^{d}$.

REMARK 4.11. For $\lambda_{0} \geq 0$, by [23, Lemma 4.6 and Lemma 4.7], the function $h$ is finely continuous and satisfies

$$
h(x)=E_{x}\left[\int_{0}^{\infty} h\left(X_{t}\right) d A_{t}^{\mu+\lambda_{0} v}\right],
$$

for all $x \in \boldsymbol{R}^{d}$.

We now show that the function $h$ is bounded. For a signed measure $\mu=\mu^{+}-\mu^{-} \in$ $\mathcal{K}_{d, \alpha}^{\infty}-\mathcal{K}_{d, \alpha}^{\infty}$, we assume that $\mathcal{H}^{\mu}$ is subcritical or critical and $h$ is a positive $\mathcal{H}^{\mu}$-harmonic function. Let $P_{t}^{\mu, h}$ be the $h$-transformed semigroup of $P_{t}^{\mu}$, i.e.,

$$
P_{t}^{\mu, h} f(x)=\int_{\{0<h<\infty\}} \frac{1}{h(x)} p^{\mu}(t, x, y) h(y) f(y) d y .
$$

$P_{t}^{\mu, h}$ is the $L^{2}\left(h^{2} m\right)$-strongly continuous symmetric Markov semigroup, $\left(P_{t}^{\mu, h} f, g\right)_{h^{2} m}=$ $\left(f, P_{t}^{\mu, h} g\right)_{h^{2} m}$. Therefore we can define a Dirichlet form $\left(\mathcal{E}^{\mu, h}, \mathcal{D}\left(\mathcal{E}^{\mu, h}\right)\right)$ generated by the semigroup $P_{t}^{\mu, h}$. Let $\mathcal{D}_{e}\left(\mathcal{E}^{\mu, h}\right)$ be an extended Dirichlet space of $\mathcal{D}\left(\mathcal{E}^{\mu, h}\right)$. Setting a space

$$
\mathcal{D}_{e}\left(\mathcal{E}^{\mu}\right)=\left\{u ; \frac{u}{h} \in \mathcal{D}_{e}\left(\mathcal{E}^{\mu, h}\right)\right\},
$$


we see that $\mathcal{E}^{\mu}(u, u)=\mathcal{E}^{\mu, h}(u / h, u / h)$ on $\mathcal{D}_{e}\left(\mathcal{E}^{\mu}\right)$ and call the form $\left(\mathcal{E}^{\mu}, \mathcal{D}_{e}\left(\mathcal{E}^{\mu}\right)\right)$ an extended Schrödinger form [23]. We are able to identify $\mathcal{D}_{e}\left(\mathcal{E}^{\mu}\right)$ with the family of $m$ measurable functions $u$ on $\boldsymbol{R}^{d}$ such that $|u|<\infty m$-a.e. and there exists an $\mathcal{E}^{\mu}$-Cauchy sequence $\left\{u_{n}\right\} \subset \mathcal{D}_{e}\left(\mathcal{E}^{\mu}\right)$ such that $\lim _{n \rightarrow \infty} u_{n}=u m$-a.e. This is said to be an approximating sequence for $u \in \mathcal{D}_{e}\left(\mathcal{E}^{\mu}\right)$. Then, for $u \in \mathcal{D}_{e}\left(\mathcal{E}^{\mu}\right)$ and its approximating sequence $\left\{u_{n}\right\}$,

$$
\mathcal{E}^{\mu}(u, u)=\lim _{n \rightarrow \infty} \mathcal{E}^{\mu}\left(u_{n}, u_{n}\right), u \in \mathcal{D}_{e}\left(\mathcal{E}^{\mu}\right) .
$$

Generally, if $\left(\mathcal{E}^{\mu}, \mathcal{D}_{e}\left(\mathcal{E}^{(\alpha)}\right)\right)$ is a subcritical Schrödinger form, that is, the associated operator $\mathcal{H}^{\mu}$ is subcritical, then $\left(\mathcal{E}^{\mu}, \mathcal{D}_{e}\left(\mathcal{E}^{\mu}\right)\right)$ becomes a Hilbert space by [10, Theorem 1.5.5]. If $\left(\mathcal{E}^{\mu}, \mathcal{D}_{e}\left(\mathcal{E}^{(\alpha)}\right)\right)$ is a critical Schrödinger form then its ground state $h$ belongs to $\mathcal{D}_{e}\left(\mathcal{E}^{\mu}\right)$ on account of [10, Theorem 1.6.3]. We see from Theorem 2.2 that,

$$
\mathcal{E}^{\mu}(u, u)=\mathcal{E}^{(\alpha)}(u, u)-\int_{\boldsymbol{R}^{d}} u^{2} d \mu \leq\left(1+\|G|\mu|\|_{\infty}\right) \mathcal{E}^{(\alpha)}(u, u),
$$

thus $\mathcal{D}_{e}\left(\mathcal{E}^{(\alpha)}\right) \subset \mathcal{D}_{e}\left(\mathcal{E}^{\mu}\right)$.

By Lemma 4.2, the Schrödinger type operator is not subcritical. Let $w \not \equiv 0$ be a nonnegative bounded Borel function with compact support, and define $\eta_{w}=-\mu-\lambda_{0} v+w d x$. Denote

$$
\mathcal{H}^{\eta_{w}}=\frac{1}{2}(-\Delta)^{\alpha / 2}+\eta_{w}
$$

Then, since $\mathcal{H}^{\eta_{w}}$ is subcritical, we know that

(4.11) $\inf \left\{\mathcal{E}^{(\alpha)}(u, u)-\lambda_{0} \int_{\boldsymbol{R}^{d}} u^{2} d v+\int_{\boldsymbol{R}^{d}} u^{2} w d x ; u \in \mathcal{D}\left(\mathcal{E}^{(\alpha)}\right), \int_{\boldsymbol{R}^{d}} u^{2} d \mu=1\right\}>1$.

The operator $\mathcal{H}^{\eta_{w}}$ has a Green function $G^{\eta_{w}}(x, y)$ with

$$
0<c G(x, y) \leq G^{\eta_{w}}(x, y) \leq C G(x, y), x \neq y .
$$

Let $G^{\eta_{w}}$ be the Green operator, $G^{\eta_{w}} f(x)=\int_{\boldsymbol{R}^{d}} G^{\eta_{w}}(x, y) f(y) d y$.

LEMMA 4.12. For a non-negative function $\varphi \in C_{0}\left(\boldsymbol{R}^{d}\right), G^{\eta_{w}} \varphi$ is in $\mathcal{D}_{e}\left(\mathcal{E}^{\eta_{w}}\right)$.

Proof. Let $G_{\beta}^{\eta_{w}}$ be a $\beta$-resolvent of $\mathcal{H}^{\eta_{w}}$. Then $G_{\beta}^{\eta_{w}} \varphi$ belongs to $\mathcal{D}_{e}\left(\mathcal{E}^{(\alpha)}\right)$ and $G_{\beta}^{\eta_{w}} \varphi \uparrow$ $G^{\eta_{w}} \varphi$ as $\beta \rightarrow 0$. We see from the inequality (4.12) that

$$
\begin{aligned}
\mathcal{E}_{\beta}^{\eta_{w}}\left(G_{\beta}^{\eta_{w}} \varphi, G_{\beta}^{\eta_{w}} \varphi\right) & =\left(\left(\mathcal{H}^{\eta_{w}}+\beta\right) G_{\beta}^{\eta_{w}} \varphi, G_{\beta}^{\eta_{w}} \varphi\right) \\
& =\left(\varphi, G_{\beta}^{\eta_{w}} \varphi\right) \leq\left(\varphi, G^{\eta_{w}} \varphi\right) \leq C(\varphi, G \varphi)<\infty .
\end{aligned}
$$

This implies $G_{\beta}^{\eta_{w}} \varphi$ approximates $G^{\eta_{w}} \varphi$ in $\mathcal{E}^{\eta_{w}}$-norm, which shows $G^{\eta_{w}} \varphi \in \mathcal{D}_{e}\left(\mathcal{E}^{\eta_{w}}\right)$.

LEMMA 4.13. For any non-negative $\varphi \in C_{0}\left(\boldsymbol{R}^{d}\right)$,

$$
\mathcal{E}^{\eta_{w}}\left(G^{\eta_{w}} \varphi, f\right)=\int_{\boldsymbol{R}^{d}} \varphi f d x, \quad f \in \mathcal{D}_{e}\left(\mathcal{E}^{\eta_{w}}\right) .
$$


REMARK 4.14. If $\mathcal{H}^{\eta_{w}}$ is subcritical, then the measure $\eta_{w}$ is gaugeable, that is, $\sup _{x \in \boldsymbol{R}^{d}} E_{x}\left[e^{A_{\infty}^{\eta_{w}}}\right]<\infty\left[24\right.$, Theorem 3.1]. Furthermore $h_{0}(x)=E_{x}\left[e^{A_{\infty}^{\eta_{w}}}\right]$ is $\mathcal{H}^{\eta_{w}}$-harmonic (see [23]). In fact, we consider $A_{\infty}^{\eta_{w}}=A_{\tau_{D}}^{\eta_{w}}+A_{\infty}^{\eta_{w}} \circ \theta_{\tau_{D}}$ and then

$$
\begin{aligned}
& h_{0}(x)=E_{x}\left[e^{A_{\infty}^{\eta_{w}}}\right]=E_{x}\left[e^{A_{\tau_{D}}^{\eta_{w}}} e^{A_{\infty}^{\eta_{w} \circ \theta_{\tau_{D}}}}\right] \\
& =E_{x}\left[e^{A_{\tau_{D}}^{\eta_{w}}} E_{x}\left[e^{A_{\infty}^{\eta_{w} \circ \theta_{\tau_{D}}}} \mid \mathcal{F}_{\tau_{D}}\right]\right] \\
& =E_{x}\left[e^{A_{\tau}^{\eta_{D}} E_{X_{\tau_{D}}}}\left[e^{A_{\infty}^{\eta_{w}}}\right]\right] \\
& =E_{x}\left[e^{A_{\tau}^{\eta_{D} w}} h_{0}\left(X_{\tau_{D}}\right)\right], \quad x \in D,
\end{aligned}
$$

where $D$ is a bounded domain.

ProOF OF LEMMA 4.13. We consider $h$-transformed semigroup $P_{t}^{\eta_{w}, h_{0}}$ of $P_{t}^{\eta_{w}}$. Let $\left(\mathcal{E}^{\eta_{w}, h_{0}}, \mathcal{D}^{\eta_{w}, h_{0}}\right)$ be the Dirichlet form generated by $P_{t}^{\eta_{w}, h_{0}}$ and denote by $G^{\eta_{w}, h_{0}}$ its Green operator, i.e.,

$$
G^{\eta_{w}, h_{0}} f=\frac{1}{h_{0}} G^{\eta_{w}}\left(h_{0} \cdot f\right) .
$$

Then, for a non-negative function $\varphi \in C_{0}\left(\boldsymbol{R}^{d}\right)$,

$$
\int_{\boldsymbol{R}^{d}} G^{\eta_{w}, h_{0}}\left(\frac{\varphi}{h_{0}}\right) \cdot \frac{\varphi}{h_{0}} h_{0}^{2} d x=\int_{\boldsymbol{R}^{d}} G^{\eta_{w}} \varphi \cdot \varphi d x \leq C \int_{\boldsymbol{R}^{d}} G \varphi \cdot \varphi d x .
$$

Thus [10, Theorem 1.5.4] leads us to that $G^{\eta_{w}, h_{0}}\left(\varphi / h_{0}\right)$ belongs to $\mathcal{D}_{e}\left(\mathcal{E}^{\eta_{w}, h_{0}}\right)$ and for any $f \in \mathcal{D}_{e}\left(\mathcal{E}^{\eta_{w}}\right)$,

$$
\mathcal{E}^{\eta_{w}, h_{0}}\left(G^{\eta_{w}, h_{0}}\left(\frac{\varphi}{h_{0}}\right), \frac{f}{h_{0}}\right)=\int_{\boldsymbol{R}^{d}} \varphi f d x
$$

Noting that

$$
\mathcal{E}^{\eta_{w}, h_{0}}\left(G^{\eta_{w}, h_{0}}\left(\frac{\varphi}{h_{0}}\right), \frac{f}{h_{0}}\right)=\mathcal{E}^{\eta_{w}, h_{0}}\left(\frac{G^{\eta_{w}} \varphi}{h_{0}}, \frac{f}{h_{0}}\right)=\mathcal{E}^{\eta_{w}}\left(G^{\eta_{w}} \varphi, f\right),
$$

we have Lemma 4.13 .

The next theorem was first obtained by Murata [13, Theorem 2.2] for $\alpha=2$, and Takeda and Tsuchida [23, Theorem 4.8] extended to symmetric $\alpha$-stable processes. In [23, Theorem 4.8], the constant $\lambda_{0}$ is not negative. We extend [23, Theorem 4.8] for $\lambda_{0}<0$.

THEOREM 4.15. For $w \in C_{0}\left(\boldsymbol{R}^{d}\right)$ with $w \geq 0, w \neq \equiv$, let $\eta_{w}=-\mu-\lambda_{0} v+w d x$. The function $h$ defined in (4.7) satisfies

$$
h(x)=\int_{\boldsymbol{R}^{d}} G^{\eta_{w}}(x, y) h(y) w(y) d y .
$$

ProOF. Because of Lemma 4.12,

$$
\varphi \in C_{0}\left(\boldsymbol{R}^{d}\right) \Longrightarrow G_{\beta}^{\eta_{w}} \varphi \in \mathcal{D}_{e}\left(\mathcal{E}^{(\alpha)}\right), \quad \beta>0 .
$$


By the equation (4.6),

$$
\mathcal{E}^{(\alpha)}\left(h, G_{\beta}^{\eta_{w}} \varphi\right)-\int_{\boldsymbol{R}^{d}} h G_{\beta}^{\eta_{w}} \varphi d \mu-\lambda_{0} \int_{\boldsymbol{R}^{d}} h G_{\beta}^{\eta_{w}} \varphi d \nu=0 .
$$

Thus,

$$
\begin{aligned}
\mathcal{E}^{\eta_{w}}\left(h, G_{\beta}^{\eta_{w}} \varphi\right) & \\
= & \mathcal{E}^{(\alpha)}\left(h, G_{\beta}^{\eta_{w}} \varphi\right)-\int_{\boldsymbol{R}^{d}} h G_{\beta}^{\eta_{w}} \varphi d \mu-\lambda_{0} \int_{\boldsymbol{R}^{d}} h G_{\beta}^{\eta_{w}} \varphi d v+\int_{\boldsymbol{R}^{d}} h G_{\beta}^{\eta_{w}} \varphi \cdot w d x \\
& =\int_{\boldsymbol{R}^{d}} h G_{\beta}^{\eta_{w}} \varphi \cdot w d x=\int_{\boldsymbol{R}^{d}} G_{\beta}^{\eta_{w}}(h w) \cdot \varphi d x .
\end{aligned}
$$

The last equation follows from the symmetry of $G_{\beta}^{\eta_{w}}$. As $\beta \rightarrow 0$,

$$
\mathcal{E}^{\eta_{w}}\left(h, G^{\eta_{w}} \varphi\right)=\int_{\boldsymbol{R}^{d}} G^{\eta_{w}}(h w) \varphi d x .
$$

On the other hand, Lemma 4.13 tells us

$$
\mathcal{E}^{\eta_{w}}\left(h, G^{\eta_{w}} \varphi\right)=\int_{\boldsymbol{R}^{d}} h \varphi d x, \text { for all } \varphi \in \mathcal{D}_{e}\left(\mathcal{E}^{\eta_{w}}\right) .
$$

Comparing equations (4.15) with (4.16), we have

$$
\begin{aligned}
h(x) & =G^{\eta_{w}}(h w)(x) \\
& =\int_{\boldsymbol{R}^{d}} G^{\eta_{w}}(x, y) h(y) w(y) d y, \text { m-a.e. }
\end{aligned}
$$

By the same argument as in Lemma 4.10, we are able to refine from " $m$-a.e. $x$ " to "any $x$ ".

We now show the boundedness of $h$.

LEMMA 4.16. The function $h$ is bounded.

Proof. Because of the fine continuity of $h$, there exists a compact set $K$ such that $h(x) \leq c$ for any $x \in K$. Let $\eta_{K}=-\mu-v+I_{K}(x) d x$. Then Theorem 4.15 implies

$$
h(x)=\int_{\boldsymbol{R}^{d}} G^{\eta_{K}}(x, y) h(y) I_{K}(y) d y .
$$

Since $G^{\eta_{K}}(x, y)$ is equivalent to $G(x, y)$ by (4.12) and $h$ is bounded on $K$, it holds that

$$
h(x) \leq c \int_{\boldsymbol{R}^{d}} G^{\eta_{K}}(x, y) I_{K}(y) d y \leq C\left\|G\left(I_{K} m\right)\right\|_{\infty}<\infty .
$$

The last inequality derives from $I_{K} m \in \mathcal{K}_{d, \alpha}^{\infty}$.

REMARK 4.17. For $\lambda_{0} \geq 0$, we see from [23, Lemma 4.9] that the function $h$ is bounded.

LEMMA 4.18. The function $h$ is $P_{t}^{\mu+\lambda_{0} v}$-excessive. That is

$$
E_{x}\left[\exp \left(A_{t}^{\mu}+\lambda_{0} A_{t}^{v}\right) h\left(X_{t}\right)\right] \leq h(x) .
$$


Proof. Put

$$
N_{t}=E_{x}\left[\int_{0}^{\infty} \exp \left(\lambda_{0} A_{s}^{v}\right) u_{0}\left(X_{s}\right) d A_{s}^{\mu} \mid \mathcal{F}_{t}\right] .
$$

Then we see from (4.8) that

$$
\exp \left(\lambda_{0} A_{t}^{v}\right) h\left(X_{t}\right)=N_{t}-\int_{0}^{t} \exp \left(\lambda_{0} A_{s}^{v}\right) u_{0}\left(X_{s}\right) d A_{s}^{\mu} .
$$

By Itō's formula,

$$
\begin{aligned}
\exp \left(A_{t}^{\mu}\right. & \left.+\lambda_{0} A_{t}^{v}\right) h\left(X_{t}\right)=\exp \left(A_{t}^{\mu}\right) \exp \left(\lambda_{0} A_{t}^{v}\right) h\left(X_{t}\right) \\
= & \exp \left(A_{t}^{\mu}\right)\left\{N_{t}-\int_{0}^{t} \exp \left(\lambda_{0} A_{s}^{v}\right) h\left(X_{s}\right) d A_{s}^{\mu}\right\} \\
= & h\left(X_{0}\right)+\int_{0}^{t} \exp \left(A_{s}^{\mu}\right)\left(d N_{s}-\exp \left(\lambda_{0} A_{s}^{\mu}\right) h\left(X_{s}\right) d A_{s}^{\mu}\right) \\
& +\int_{0}^{t} \exp \left(A_{s}^{\mu}\right) \exp \left(\lambda_{0} A_{s}^{\mu}\right) h\left(X_{s}\right) d A_{s}^{\mu} \\
= & h\left(X_{0}\right)+\int_{0}^{t} \exp \left(A_{s}^{\mu}\right) d N_{s} .
\end{aligned}
$$

Here $N_{t}$ is martingale, so is the second term of the right-hand side. Taking the expectations, we have

$$
E_{x}\left[\exp \left(A_{t}^{\mu}+\lambda_{0} A_{t}^{v}\right) h\left(X_{t}\right)\right] \leq h(x)
$$

REMARK 4.19. For $\lambda_{0} \geq 0$, we see from [23, Lemma 4.10] that the function $h$ is excessive.

We see from Lemma 4.18 that $h$-transformed semigroup $P_{t}^{\mu+\lambda_{0} v, h}$ generates an $h^{2} m$ symmetric Markov process. Let us denote by $\boldsymbol{M}^{\mu+\lambda_{0} v, h}$ the Markov process generated by $P_{t}^{\mu+\lambda_{0} \nu, h}$. Then $\boldsymbol{M}^{\mu+\lambda_{0} v, h}$ is recurrent because of non-subcriticality of $\mathcal{H}^{\mu+\lambda_{0} v}$, in particular, conservative, $P_{t}^{\mu+\lambda_{0} v, h} 1=1$. As a result, the function $h$ is $P_{t}^{\mu+\lambda_{0} v}$-invariant, i.e.,

$$
P_{t}^{\mu+\lambda_{0} v} h=h .
$$

LEMMA 4.20. A finely continuous $P_{t}^{\mu+\lambda_{0} v}$-excessive function is unique up to multiplication.

Proof. Let $h, h^{\prime}$ be $P_{t}^{\mu+\lambda_{0} v}$-excessive functions. By the excessivity, $P_{t}^{\mu+\lambda_{0} v} h^{\prime}(x) \uparrow$ $h^{\prime}(x)$ and the function $h(x)$ is strictly positive for all $x$,

$$
E_{x}\left[\exp \left(A_{t}^{\mu+\lambda_{0} v}\right) h\left(X_{t}\right) \frac{h^{\prime}}{h}\left(X_{t}\right)\right] \leq h \cdot \frac{h^{\prime}}{h}(x), \quad \text { for all } t>0 .
$$

Thus

$$
\frac{h^{\prime}}{h}(x) \geq \frac{1}{h(x)} E_{x}\left[\exp \left(A_{t}^{\mu+\lambda_{0} v}\right) h\left(X_{t}\right) \frac{h^{\prime}}{h}\left(X_{t}\right)\right]
$$




$$
=E_{x}^{\mu+\lambda_{0} v, h}\left[\frac{h^{\prime}}{h}\left(X_{t}\right)\right] .
$$

For $y \in \boldsymbol{R}^{d}$ and $\varepsilon_{n} \rightarrow 0$ as $n \rightarrow \infty, \sigma_{B\left(y, \varepsilon_{n}\right)}<\infty, P_{x}^{\mu+\lambda_{0} v, h}$-a.s. by [10, Problem 4.6.3], where $B\left(y, \varepsilon_{n}\right)=\left\{z ;|z-y|<\varepsilon_{n}\right\}$. Denote $\sigma_{n}=\sigma_{B\left(y, \varepsilon_{n}\right)}$. Replacing $t$ by $\sigma_{n}$,

$$
E_{x}^{\mu+\lambda_{0} v, h}\left[\frac{h^{\prime}}{h}\left(X_{\sigma_{n}}\right)\right] \leq \frac{h^{\prime}}{h}(x),
$$

and taking $n \rightarrow \infty$, we see that the left-hand side converges to $h^{\prime} / h(y)$. By Fatou's lemma, we obtain

$$
\begin{aligned}
\frac{h^{\prime}}{h}(y) & =E_{x}^{\mu+\lambda_{0} v, h}\left[\liminf _{n \rightarrow \infty} \frac{h^{\prime}}{h}\left(X_{\sigma_{n}}\right)\right] \\
& \leq \liminf _{n \rightarrow \infty} E_{x}^{\mu+\lambda_{0} v, h}\left[\frac{h^{\prime}}{h}\left(X_{\sigma_{n}}\right)\right] \\
& \leq \frac{h^{\prime}}{h}(x),
\end{aligned}
$$

for any $x, y$. Thus we see also $h^{\prime}(x) / h(x) \leq h^{\prime}(y) / h(y)$. Setting $c=h^{\prime}(x) / h(x)$ for a fixed $x \in \boldsymbol{R}^{d}$, we have $h^{\prime}(y)=\operatorname{ch}(y)$ for every $y \in \boldsymbol{R}^{d}$.

Proposition 4.21. The function $h$ is $\mathcal{H}^{\mu+\lambda_{0} v}$-harmonic, i.e., for any bounded domain $D$,

$$
E_{x}\left[\exp \left(A_{\tau_{D}}^{\mu+\lambda_{0} v}\right) h\left(X_{\tau_{D}}\right)\right]=h(x), x \in D
$$

ProOF. Set

$$
M_{t}=\exp \left(A_{t}^{\mu+\lambda_{0} v}\right) h\left(X_{t}\right)
$$

Then $M_{t}$ is a martingale. In fact, by the additivity $A_{t}-A_{s}=A_{t-s}\left(\theta_{s}\right)$ for any $0<s<t$ and the Markov property, we obtain

$$
\begin{aligned}
E_{x}\left[M_{t} \mid \mathcal{F}_{s}\right] & =E_{x}\left[\exp \left(A_{t}^{\mu+\lambda_{0} v}\right) h\left(X_{t}\right) \mid \mathcal{F}_{s}\right] \\
& =E_{x}\left[\exp \left(A_{s}^{\mu+\lambda_{0} v}\right) \exp \left(A_{t}^{\mu+\lambda_{0} v}-A_{s}^{\mu+\lambda_{0} v}\right) h\left(X_{t}\right) \mid \mathcal{F}_{s}\right] \\
& =\exp \left(A_{s}^{\mu+\lambda_{0} v}\right) E_{x}\left[\exp \left(A_{t-s}^{\mu+\lambda_{0} v}\left(\theta_{s}\right)\right) h\left(X_{t}\right) \mid \mathcal{F}_{s}\right] \\
& =\exp \left(A_{s}^{\mu+\lambda_{0} v}\right) E_{X_{s}}\left[\exp \left(A_{t-s}^{\mu+\lambda_{0} v}\right) h\left(X_{t-s}\right)\right] .
\end{aligned}
$$

We see from the equality (4.18) that

$$
\exp \left(A_{s}^{\mu+\lambda_{0} v}\right) P_{t-s}^{\mu+\lambda_{0} v} h\left(X_{s}\right)=\exp \left(A_{s}^{\mu+\lambda_{0} v}\right) h\left(X_{s}\right)=M_{s} .
$$

That is, $E_{x}\left[M_{t} \mid \mathcal{F}_{s}\right]=M_{s}$ for any $0<s<t$.

On account of the optional stopping theorem, for a bounded domain $D$ of $\boldsymbol{R}^{d}$

$$
E_{x}\left[\exp \left(A_{t \wedge \tau_{D}}^{\mu+\lambda_{0} v}\right) h\left(X_{t \wedge \tau_{D}}\right)\right]=h(x) .
$$

We now show the following claim

$$
\inf \left\{\mathcal{E}^{(\alpha)}(u, u)-\int_{\boldsymbol{R}^{d}} u^{2} d \mu-\lambda_{0} \int_{\boldsymbol{R}^{d}} u^{2} d v ; u \in \mathcal{D}\left(\mathcal{E}_{D}^{(\alpha)}\right), \int_{\boldsymbol{R}^{d}} u^{2} d x=1\right\}>0
$$


where $\mathcal{E}_{D}^{(\alpha)}$ is the part Dirichlet form of $\mathcal{E}^{(\alpha)}$ (see [10, Section 4.4]). In fact, if the left-hand side is equal to zero, then there exists a function $u_{*} \in \mathcal{D}\left(\mathcal{E}_{D}^{(\alpha)}\right)$ such that

$$
\mathcal{E}^{(\alpha)}\left(u_{*}, u_{*}\right)-\lambda_{0} \int_{\boldsymbol{R}^{d}} u_{*}^{2} d v=\int_{\boldsymbol{R}^{d}} u_{*}^{2} d \mu .
$$

Note that $\int_{\boldsymbol{R}^{d}} u_{*}^{2} d \mu>0$ because of $u_{*}>0$ q.e. on $D$ and $\mu(D)>0$. Then the function $\tilde{u}_{0}=u_{*} / \sqrt{\int_{\boldsymbol{R}^{d}} u_{*}^{2} d \mu}$ attains the infimum of (4.2), and thus $u_{*}>0$ q.e. on $\boldsymbol{R}^{d}$ as same argument as in Lemma 4.10 for the function $u_{0}$. However, this contradicts $u_{*}=0 \mathrm{~m}$-a.e. on $\boldsymbol{R}^{d} \backslash D$.

From [23, Lemma 2.2], we see that the equation (4.20) implies

$$
\inf \left\{\mathcal{E}^{(\alpha)}(u, u)-\lambda_{0} \int_{\boldsymbol{R}^{d}} u^{2} d \nu ; u \in \mathcal{D}\left(\mathcal{E}_{D}^{(\alpha)}\right), \int_{\boldsymbol{R}^{d}} u^{2} d \mu=1\right\}>1,
$$

and then $\mu+\lambda_{0} v$ is gaugeable on $D$ by [24, Theorem 3.1], that is,

$$
\sup _{x \in D} E_{x}\left[\exp \left(A_{\tau_{D}}^{\mu+\lambda_{0} v}\right)\right]<\infty .
$$

We see from [5, Corollary 2.9] that

$$
\sup _{x \in D} E_{x}\left[\sup _{0 \leq t \leq \tau_{D}} \exp \left(A_{t}^{\mu+\lambda_{0} v}\right)\right]<\infty .
$$

Noting that

$$
\left|\exp \left(A_{t \wedge \tau_{D}}^{\mu+\lambda_{0} v}\right) h\left(X_{t \wedge \tau_{D}}\right)\right| \leq\|h\|_{\infty} \sup _{0 \leq t \leq \tau_{D}} \exp \left(A_{t}^{\mu+\lambda_{0} v}\right)<\infty,
$$

we have from the quasi-left continuity of $\boldsymbol{M}^{\alpha}$ and the bounded convergence theorem

$$
\begin{aligned}
\lim _{t \rightarrow \infty} E_{x}\left[\exp \left(A_{t \wedge \tau_{D}}^{\mu+\lambda_{0} v}\right) h\left(X_{t \wedge \tau_{D}}\right)\right] & =E_{x}\left[\lim _{t \rightarrow \infty} \exp \left(A_{t \wedge \tau_{D}}^{\mu+\lambda_{0} v}\right) h\left(X_{t \wedge \tau_{D}}\right)\right] \\
& =E_{x}\left[\exp \left(A_{\tau_{D}}^{\mu+\lambda_{0} v}\right) h\left(X_{\tau_{D}}\right)\right] .
\end{aligned}
$$

The right-hand side is equal to $h(x)$ by the equation (4.19).

REMARK 4.22. For $\lambda_{0} \geq 0$, we see from [23, Proposition 4.12] that the function $h$ is $\mathcal{H}^{\mu+\lambda_{0} v}$-harmonic.

Next we show the continuity of $h$.

LEMMA 4.23. The function $h$ satisfies

$$
h(x)=E_{x}\left[h\left(X_{\tau_{D}}\right)\right]+E_{x}\left[\int_{0}^{\tau_{D}} h\left(X_{t}\right) d A_{t}^{\mu+\lambda_{0} \nu}\right] .
$$

PROOF. Since $h$ is $\mathcal{H}^{\mu+\lambda_{0} v}$-harmonic,

$$
\begin{aligned}
h\left(X_{t}\right) & =E_{X_{t}}\left[\exp \left(A_{\tau_{D}}^{\mu+\lambda_{0} v}\right) h\left(X_{\tau_{D}}\right)\right] \\
& =E_{x}\left[\exp \left(A_{\tau_{D}}^{\mu+\lambda_{0} v}\left(\theta_{t}\right)\right) h\left(X_{\tau_{D}+t}\right) \mid \mathcal{F}_{t}\right] \\
& =E_{x}\left[\exp \left(A_{\tau_{D}+t}^{\mu+\lambda_{0} v}-A_{t}^{\mu+\lambda_{0} v}\right) h\left(X_{\tau_{D}+t}\right) \mid \mathcal{F}_{t}\right]
\end{aligned}
$$




$$
\begin{aligned}
& =\exp \left(-A_{t}^{\mu+\lambda_{0} v}\right) E_{x}\left[\exp \left(A_{\tau_{D}+t}^{\mu+\lambda_{0} v}\right) h\left(X_{\tau_{D}+t}\right) \mid \mathcal{F}_{t}\right] \\
& =\exp \left(-A_{t}^{\mu+\lambda_{0} v}\right) \exp \left(A_{\tau_{D}}^{\mu+\lambda_{0} v}\right) h\left(X_{\tau_{D}}\right) .
\end{aligned}
$$

Since $M_{t}$ in Proposition 4.21 is martingale, we have the last equality. For $0 \leq t \leq \tau_{D}$, $X_{t} \in D$,

$$
\begin{aligned}
E_{x}\left[\int_{0}^{\tau_{D}} h\left(X_{t}\right) d A_{t}^{\mu+\lambda_{0} v}\right] \\
\quad=E_{x}\left[\int_{0}^{\tau_{D}} \exp \left(-A_{t}^{\mu+\lambda_{0} v}+A_{\tau_{D}}^{\mu+\lambda_{0} v}\right) h\left(X_{\tau_{D}}\right) d A_{t}^{\mu+\lambda_{0} v}\right] \\
\quad=E_{x}\left[\exp \left(A_{\tau_{D}}^{\mu+\lambda_{0} v}\right) h\left(X_{\tau_{D}}\right) \int_{0}^{\tau_{D}} \exp \left(-A_{t}^{\mu+\lambda_{0} v}\right) d A_{t}^{\mu+\lambda_{0} v}\right] \\
\quad=E_{x}\left[\exp \left(A_{\tau_{D}}^{\mu+\lambda_{0} v}\right) h\left(X_{\tau_{D}}\right)\left\{1-\exp \left(-A_{\tau_{D}}^{\mu+\lambda_{0} v}\right)\right\}\right] \\
=E_{x}\left[\exp \left(A_{\tau_{D}}^{\mu+\lambda_{0} v}\right) h\left(X_{\tau_{D}}\right)\right]-E_{x}\left[h\left(X_{\tau_{D}}\right)\right] \\
=h(x)-E_{x}\left[h\left(X_{\tau_{D}}\right)\right] .
\end{aligned}
$$

We have the next proposition by the same argument as in [4, Proposition 6.1].

PROPOSITION 4.24. The function $h$ is continuous.

We consider the asymptotic behavior of $h$ as $|x| \rightarrow \infty$. Let $w$ be a positive continuous function with compact support. Suppose that $0 \in \operatorname{supp}[w] \subset B(R)$. By Theorem 4.15, the continuity of $h$ and the strict positivity of $f$,

$$
c \int_{B(R)} G^{\eta_{w}}(x, y) w(y) d y \leq h(x) \leq C \int_{B(R)} G^{\eta_{w}}(x, y) w(y) d y,
$$

and by the Green kernel estimate (4.12),

$$
c \int_{B(R)} G(x, y) w(y) d y \leq h(x) \leq C \int_{B(R)} G(x, y) w(y) d y .
$$

The Harnack inequality to $\{G(x, \cdot)\}_{\left\{x \in B(R)^{c}\right\}}$ says that for any $x \in B(R)^{c}$ and $y \in \operatorname{supp}[w]$

$$
c G(x, y) \leq G(x, 0) \leq C G(x, y) .
$$

Therefore we see that

$$
c G(x, 0) \leq h(x) \leq C G(x, 0) \text { for } x \in B(R)^{c},
$$

namely,

$$
\frac{c}{|x|^{d-\alpha}} \leq h(x) \leq \frac{C}{|x|^{d-\alpha}} .
$$

The operator $\mathcal{H}^{\mu+\lambda_{0} v}$ is said to be positive (resp. null) critical if the ground state $h$ is in (resp. not in) $L^{2}(m)$. From the equation (4.23), we have the following. 
THEOREM 4.25. The operator $\mathcal{H}^{\mu+\lambda_{0} v}$ is null critical if and only if $\alpha<d \leq 2 \alpha$.

REMARK 4.26. For $\lambda_{0} \geq 0$, we see from [23, Theorem 4.1.5] that the function $h(x)=$ $E_{x}\left[\int_{0}^{\infty} h\left(X_{t}\right) d A_{t}^{\mu+\lambda_{0} v}\right]$ satisfies (4.23). Hence Theorem 4.25 holds for any $\lambda_{0}$.

5. An extension of Oshima's inequality. In this section, we prove a functional inequality for critical Schrödinger forms. This inequality is regarded as a version of Oshima's inequality. For $\lambda_{0} \geq 0$, Takeda and Tsuchida [23] prove it. We suppose $\lambda_{0}<0$.

LEMMA 5.1. Let $h$ be the $\mathcal{H}^{\mu+\lambda_{0} v}$-harmonic function constructed in Section 4. Then the h-transformed semigroup $P_{t}^{\mu+\lambda_{0} v, h}$ of $P_{t}^{\mu+\lambda_{0} v}$ has the strong Feller property.

PROOF. We follow the argument in [9, Corollary 5.2.7]. Let $f$ be a bounded Borel function and $\left\{x_{n}\right\}$ a sequence so that $x_{n} \rightarrow x$ as $n \rightarrow \infty$. Recall that $p^{\mu+\lambda_{0} v}(t, x, y)$ is jointly continuous [1, Theorem 3.10]. By Fatou's lemma and the continuity of $h$,

$$
\begin{array}{r}
\liminf _{n \rightarrow \infty} \int_{\boldsymbol{R}^{d}} \frac{1}{h\left(x_{n}\right)} p^{\mu+\lambda_{0} v}\left(t, x_{n}, y\right) h(y)\left(\|f\|_{\infty} \pm f(y)\right) d y \\
\geq \int_{\boldsymbol{R}^{d}} \frac{1}{h(x)} p^{\mu+\lambda_{0} v}(t, x, y) h(y)\left(\|f\|_{\infty} \pm f(y)\right) d y .
\end{array}
$$

Thus, the mapping

$$
x \mapsto \int_{\boldsymbol{R}^{d}} \frac{1}{h(x)} p^{\mu+\lambda_{0} v}(t, x, y) h(y)\left(\|f\|_{\infty} \pm f(y)\right) d y
$$

is lower semi-continuous. Note that $P^{\mu+\lambda_{0} v, h}$ is recurrent, in particular, conservative. Then

$$
\begin{aligned}
& \int_{\boldsymbol{R}^{d}} \frac{1}{h(x)} p^{\mu+\lambda_{0} v}(t, x, y) h(y) f(y) d y \\
& \quad=\int_{\boldsymbol{R}^{d}} \frac{1}{h(x)} p^{\mu+\lambda_{0} v}(t, x, y) h(y)\left(\|f\|_{\infty}+f(y)\right) d y-\|f\|_{\infty} \\
& \quad=-\int_{\boldsymbol{R}^{d}} \frac{1}{h(x)} p^{\mu+\lambda_{0} v}(t, x, y) h(y)\left(\|f\|_{\infty}-f(y)\right) d y+\|f\|_{\infty},
\end{aligned}
$$

and thus the mapping

$$
x \mapsto \int_{\boldsymbol{R}^{d}} \frac{1}{h(x)} p^{\mu+\lambda_{0} v}(t, x, y) h(y) f(y) d y
$$

is lower and upper semi-continuous.

Proposition 5.2. The h-transformed process $\boldsymbol{M}^{\mu+\lambda_{0} v, h}=\left(P_{x}^{\mu+\lambda_{0} v, h}, X_{t}\right)$ is Harris recurrent, that is, for a non-negative function $f$,

$$
\int_{0}^{\infty} f\left(X_{t}\right) d t=\infty, \quad P_{x}^{\mu+\lambda_{0} v, h} \text {-a.s. }
$$

whenever $m(\{x ; f(x)>0\})>0$. 
Proof. Set $A=\{x ; f(x)>0\}$. Since $P_{t}^{\mu+\lambda_{0} v, h}$ generates an $h^{2} m$-symmetric recurrent Markov process,

$$
P_{x}\left(\sigma_{A} \circ \theta_{n}<\infty, \forall n \geq 0\right)=1, \quad \text { for } \text { q.e. } x \in \boldsymbol{R}^{d} .
$$

by [10, Theorem 4.6.6]. Moreover, since the Markov process $\boldsymbol{M}^{\mu+\lambda_{0} v, h}$ has the transition density function

$$
\frac{p^{\lambda_{0} v}(t, x, y)}{h(x) h(y)}
$$

with respect to $h^{2} m$, (5.2) holds for all $x \in \boldsymbol{R}^{d}$ by [10, Problem 4.6.3]. Using Lemma 5.1, (5.2) and the proof of [16, Chapter X, Proposition 3.11], we see that $\boldsymbol{M}^{\mu+\lambda_{0} \nu, h}$ is Harris recurrent.

THEOREM 5.3. There exists a positive function $g \in L^{1}\left(h^{2} m\right)$ and a function $\psi \in$ $C_{0}\left(\boldsymbol{R}^{d}\right)$ with $\int_{\boldsymbol{R}^{d}} \psi h^{2} d x=1$ such that, for $u \in \mathcal{D}\left(\mathcal{E}^{\mu+\lambda_{0} v}\right)$

$$
\int_{\boldsymbol{R}^{d}}\left|u(x)-h(x) L\left(\frac{u}{h}\right)\right| g(x) h(x) d x \leq C \mathcal{E}^{\mu+\lambda_{0} v}(u, u)^{1 / 2} .
$$

Here

$$
L(u)=\int_{\boldsymbol{R}^{d}} u \psi h^{2} d x .
$$

Proof. By Proposition 5.2, we can apply Oshima's inequality in [14] to the Dirichlet form $\left(\mathcal{E}^{\mu+\lambda_{0} v, h}, \mathcal{D}\left(\mathcal{E}^{\mu+\lambda_{0} v, h}\right)\right)$; there exists a positive function $g \in L^{1}\left(h^{2} m\right)$ and a function $\psi \in C_{0}\left(\boldsymbol{R}^{d}\right)$ with $\int_{\boldsymbol{R}^{d}} \psi h^{2} d x=1$ such that, for $u \in \mathcal{D}\left(\mathcal{E}^{\mu+\lambda_{0} v, h}\right)$

$$
\int_{\boldsymbol{R}^{d}}|u(x)-L(u)| g(x) h^{2}(x) d x \leq C \mathcal{E}^{\mu+\lambda_{0} v, h}(u, u)^{1 / 2} .
$$

Substituting $u / h$ for $u$ in (5.4), we obtain (5.3).

6. Differentiability of spectral function. Before proving the differentiability of the spectral function, we recall the following result in [23, Lemma 6.1].

LEMMA 6.1 ([23, Lemma 6.1]). Let X be a locally compact separable metric space, $m$ a positive Radon measure on $X$, and $(\mathcal{E}, \mathcal{D}(\mathcal{E}))$ a regular Dirichlet form on $L^{2}(X ; m)$. Let $\left\{u_{n}\right\} \subset \mathcal{D}(\mathcal{E})$ be a sequence with $\lim _{n \rightarrow \infty} \mathcal{E}\left(u_{n}, u_{n}\right)=0$ and $\lim _{n \rightarrow \infty} u_{n}=0$ m-a.e. Then there is a subsequence $\left\{u_{n_{k}}\right\}$ such that $\lim _{k \rightarrow \infty} u_{n_{k}}=0$ q.e.

PROOF OF THEOREM 1.2. First note that for $\lambda>\lambda_{0},-C(\lambda)$ is the principal eigenvalue of the operator $\mathcal{H}^{\mu+\lambda v}$ [20, Lemma 4.3] and thus $C(\lambda)$ is differentiable by the analytic perturbation theory [11, Chapter VII]. Hence it is enough to prove the differentiability of $C(\lambda)$ at $\lambda=\lambda_{0}$. Furthermore, since $C(\lambda)$ is convex by the definition and so monotonously increasing, we have only to prove the existence of a sequence $\left\{\lambda_{n}\right\}$ with $\lambda_{n}<0$ such that $d C\left(\lambda_{n}\right) / d \lambda \downarrow 0$ as $\lambda_{n} \downarrow \lambda_{0}$.

By [11, p. 405, Chapter VII (4.44)], we have

$$
\frac{d C}{d \lambda}(\lambda)=\int_{\boldsymbol{R}^{d}} u_{\lambda}^{2} d \nu, \lambda>\lambda_{0},
$$


where $u_{\lambda}$ is the $L^{2}$-normalized eigenfunction corresponding to the eigenvalue $-C(\lambda)$, that is,

$$
-C(\lambda)=\mathcal{E}^{(\alpha)}\left(u_{\lambda}, u_{\lambda}\right)-\int_{\boldsymbol{R}^{d}} u_{\lambda}^{2} d \mu-\lambda \int_{\boldsymbol{R}^{d}} u_{\lambda}^{2} d \nu .
$$

Set $u_{n}=u_{\lambda_{n}}$ and $v_{R}(d x)=I_{B(0, R)}(x) v(d x)$ for $R>0$. By the Poincaré type inequality,

$$
\begin{aligned}
\limsup _{n \rightarrow \infty} \int_{\boldsymbol{R}^{d}} u_{n}^{2} d v & =\limsup _{n \rightarrow \infty}\left(\int_{\boldsymbol{R}^{d}} u_{n}^{2} d v_{R}+\int_{\boldsymbol{R}^{d}} u_{n}^{2} d v_{R^{c}}\right) \\
& \leq \limsup _{n \rightarrow \infty}\left(\int_{\boldsymbol{R}^{d}} u_{n}^{2} d v_{R}+\mathcal{E}^{(\alpha)}\left(u_{n}, u_{n}\right)\left\|G v_{R^{c}}\right\|_{\infty}\right) .
\end{aligned}
$$

It is enough to show an existence of some sequence such that the right-hand side converges to zero as $R \rightarrow \infty$. Firstly, we consider the second term in (6.3). By (2.7), for any $\lambda_{n} \in\left(\lambda_{0}, 0\right)$ and $\varepsilon>0$

$$
\begin{aligned}
-C\left(\lambda_{n}\right) & =\mathcal{E}^{(\alpha)}\left(u_{n}, u_{n}\right)-\int_{\boldsymbol{R}^{d}} u_{n}^{2} d \mu-\lambda_{n} \int_{\boldsymbol{R}^{d}} u_{n}^{2} d v \\
& \geq \mathcal{E}^{(\alpha)}\left(u_{n}, u_{n}\right)-\int_{\boldsymbol{R}^{d}} u_{n}^{2} d \mu \\
& \geq \mathcal{E}^{(\alpha)}\left(u_{n}, u_{n}\right)-\left(\varepsilon \mathcal{E}^{(\alpha)}\left(u_{n}, u_{n}\right)+M(\varepsilon)\right) .
\end{aligned}
$$

Therefore, we have

$$
\mathcal{E}^{(\alpha)}\left(u_{n}, u_{n}\right) \leq \frac{-C\left(\lambda_{n}\right)+M(\varepsilon)}{1-\varepsilon}, \quad \text { for all } 0<\varepsilon<1 .
$$

Since the function $C(\lambda)$ is continuous and $C\left(\lambda_{0}\right)=0$,

$$
\limsup _{n \rightarrow \infty} \mathcal{E}^{(\alpha)}\left(u_{n}, u_{n}\right) \leq \frac{M(\varepsilon)}{1-\varepsilon}<\infty .
$$

We see that the second term in (6.3) converges to zero by letting $R \rightarrow \infty$.

On account of (2.5) and (6.5),

$$
\begin{aligned}
\left|\mathcal{E}^{\mu+\lambda_{0} v}\left(u_{n}, u_{n}\right)+C\left(\lambda_{n}\right)\right| & =\left|\mathcal{E}^{\mu+\lambda_{0} v}\left(u_{n}, u_{n}\right)-\mathcal{E}^{\mu+\lambda_{n} v}\left(u_{n}, u_{n}\right)\right| \\
& \leq\left(\lambda_{n}-\lambda_{0}\right) \int_{\boldsymbol{R}^{d}} u_{n}^{2} d v \\
& \leq\left(\lambda_{n}-\lambda_{0}\right)\|G \nu\|_{\infty} \mathcal{E}^{(\alpha)}\left(u_{n}, u_{n}\right) \\
& \rightarrow 0, \text { as } n \rightarrow \infty .
\end{aligned}
$$

Thus we see that

$$
\mathcal{E}^{\mu+\lambda_{0} v}\left(u_{n}, u_{n}\right) \rightarrow 0 \text { as } n \rightarrow \infty .
$$

Let $h$ be the $\mathcal{H}^{\mu+\lambda_{0} v}$-harmonic function constructed in Section 4. We denote the Dirichlet form of the $h$-transformed process by $\left(\mathcal{E}^{\mu+\lambda_{0} v, h}, \mathcal{D}\left(\mathcal{E}^{\mu+\lambda_{0} v, h}\right)\right)$. Then the equation (6.6) proves

$$
\lim _{n \rightarrow \infty} \mathcal{E}^{\mu+\lambda_{0} v, h}\left(\frac{u_{n}}{h}, \frac{u_{n}}{h}\right)=0 .
$$


Let $\psi$ and $L(u)$ be the same as in Theorem 5.3. Then since

$$
\begin{aligned}
\left|L\left(\frac{u_{n}}{h}\right)\right| & =\int_{\boldsymbol{R}^{d}} u_{n}(x) \psi(x) h(x) d x \\
& \leq \sqrt{\int_{\boldsymbol{R}^{d}} u_{n}^{2}(x) d x} \sqrt{\int_{\boldsymbol{R}^{d}} \psi^{2}(x) h^{2}(x) d x}<\infty,
\end{aligned}
$$

we may assume that $L\left(u_{n} / h\right)$ converges to a certain constant $C$ by taking a subsequence of $\left\{\lambda_{n}\right\}$ if necessary. In addition, since (5.3) says

$$
\begin{aligned}
\int_{\boldsymbol{R}^{d}} \mid & u_{n}-C h \mid g h d x \\
& \leq \int_{\boldsymbol{R}^{d}}\left|u_{n}-h L\left(\frac{u_{n}}{h}\right)\right| g h d x+\int_{\boldsymbol{R}^{d}}\left|h L\left(\frac{u_{n}}{h}\right)-C h\right| g h d x \\
& \leq C \mathcal{E}^{\mu+\lambda_{0} v}\left(u_{n}, u_{n}\right)^{1 / 2}+\int_{\boldsymbol{R}^{d}}\left|L\left(\frac{u_{n}}{h}\right)-C\right| g h^{2} d x \\
& \rightarrow 0
\end{aligned}
$$

we assume that $u_{n} \rightarrow C h m$-a.e. Now recall that $\mathcal{H}^{\mu+\lambda_{0} v}$ is null critical if and only if $\alpha<$ $d \leq 2 \alpha$. Then the constant $C$ must be equal to 0 since $h \notin L^{2}\left(\boldsymbol{R}^{d}\right)$,

$$
1=\liminf _{n \rightarrow \infty} \int_{\boldsymbol{R}^{d}} u_{n}^{2} d x \geq \int_{\boldsymbol{R}^{d}} \liminf _{n \rightarrow \infty} u_{n}^{2} d x=C^{2} \int_{\boldsymbol{R}^{d}} h^{2} d x,
$$

and consequently

$$
\lim _{n \rightarrow \infty} u_{n}=0, m \text {-a.e. }
$$

Notice that $\mathcal{E}^{\mu+\lambda_{0} v, h}$-q.e. is equivalent to $\mathcal{E}^{(\alpha)}$-q.e. Then combing (6.8), (6.9) with Lemma 6.1, we may assume that $u_{n}$ converges to 0 q.e.

Since $u_{\lambda_{n}}$ is the eigenfunction corresponding to $C\left(\lambda_{n}\right)$,

$$
u_{n}=e^{-C\left(\lambda_{n}\right) t} P_{t}^{\mu+\lambda_{n} v} u_{n}
$$

and

$$
\left\|u_{n}\right\|_{\infty}=e^{-C\left(\lambda_{n}\right) t}\left\|P_{t}^{\mu+\lambda_{n} v} u_{n}\right\|_{\infty} \leq\left\|P_{t}^{\mu+\lambda_{n} v}\right\|_{2, \infty} \leq\left\|P_{t}^{\mu}\right\|_{2, \infty}<\infty
$$

by [1, Theorem 6.1 (iii)]. From (6.3), (6.5) and the dominated convergence theorem, we see that

$$
\limsup _{n \rightarrow \infty} \int_{\boldsymbol{R}^{d}} u_{n}^{2} d \nu \leq\left\|G v_{R^{c}}\right\|_{\infty} \frac{M(\varepsilon)}{1-\varepsilon} .
$$

By letting $R \rightarrow \infty$, we complete the proof.

By the Gärtner-Ellis theorem, we have Theorem 1.1. 


\section{REFERENCES}

[ 1] S. Albeverio, P. Blanchard and Z. M. MA, Feynman-Kac semigroup in terms of signed smooth measures, in Random Partial Differential Equations, Internat. Ser. Numer. Math. 102 (1991), 1-31.

[2] K. S. Alexander and V. Sidoravicius, Pinning polymers and interfaces by random potentials, Ann. Appl. Probab. 16 (2006), 636-669.

[3] W. ARENDT AND C. J. K. BATTY, The spectral function and principal eigenvalues for Schrödinger operators, Potential Anal. 7 (1997), 415-436.

[ 4 ] K. Bogdan And T. ByczKows Ki, Potential theory of Schrödinger operator based on fractional Laplacian, Probab. Math. Statist. 20 (2000), 293-335.

[ 5 ] Z.-Q. Chen, Gaugeability and conditional gaugeability, Trans. Amer. Math. Soc. 354 (2002), 4639-4679.

[6] M. Cranston, L. Koralov, S. Molchanov and B Vainberg, Continuous model for homopolymers, J. Funct. Anal. 256 (2009), 2656-2696.

[ 7 ] M. Cranston and S. Molchanov, On phase transitions and limit theorems for homopolymers, Probability and mathematical physics, 97-112, CRM Proc. Lecture Notes 42, Amer. Math. Soc., Providence, RI, 2007.

[ 8 ] E. B. Davis, One parameter Semigroup, London Math. Soc. Monogr. 15, Academic Press, 1980.

[9] E. B. DAVIS, Heat kernels and spectral theory, Cambridge Tracs in Math. 92, Cambridge University Press, Cambridge, U.K., 1989.

[10] M. Fukushima, Y. Oshima and M. Takeda, Dirichlet forms and symmetric Markov processes, de Gruyter Stud. Math. 19, Walter de Gruyter \& Co., Berlin, 1994.

[11] T. KaTO, Perturbation theory for linear operators, Springer-Verlag, Berlin-Heidelberg-New York, 1984.

[12] K. Kuwae and M. Takahashi, Kato class measures of symmetric Markov processes under heat kernel estimates, J. Funct. Anal. 250 (2007), 86-113.

[13] M. MuratA, Structure of positive solutions to $(-\Delta+V) u=0$ in $R^{n}$, Duke Math. J. 53 (1986), 869-943.

[14] Y. Oshima, Potential of recurrent symmetric Markov processes and its associated Dirichlet spaces, in "Functional Analysis in Markov process" (ed. M. Fukushima), Lecture Notes in Math. 923 (1982), 260-275.

[15] R. G. PINS KY, Positive harmonic functions and diffusion, Cambridge Stud. Adv. Math. 45, Cambridge University Press, Cambridge, 1995.

[16] D. RevuZ And M. Yor, Continuous martingales and Brownian motion, 3rd ed., Springer-Verlag, Berlin-New York, 1998.

[17] Y. SHIOZAWA, Exponential growth of the numbers of particles for branching symmetric $\alpha$-stable processes, J. Math. Soc. Japan 60 (2008), 75-116.

[18] B. Simon, Brownian motion, $L^{p}$ properties of Schrödinger semigroups and the localization of binding, J. Funct. Anal. 35 (1998), 215-229.

[19] P. Stollmann And J. Viogt, Perturbation of Dirichlet forms by measures, Potential Anal. 5 (1998), 109138.

[20] M. TAKeDA, Large deviation principle for additive functionals of Brownian motion corresponding to Kato measures, Potential Anal. 19 (2003), 51-67.

[21] M. TAKEDA, $L^{p}$-independence of spectral bounds of Schrödinger type semigroups, J. Funct. Anal. 252 (2007), 550-565.

[22] M. TAKedA, Large deviations for additive functionals of symmetric stable processes, J. Theoret. Probab. 21 (2008), 336-355.

[23] M. TAKEDA AND K. TsuchidA, Differentiability of spectral functions for symmetric $\alpha$-stable processes, Trans. Amer. Math. Soc. 359 (2007), 4031-4054.

[24] M. TAKeda And T. Uemura, Subcriticality and gaugeablity for symmetric $\alpha$-stable processes, Forum Math. 16 (2004), 505-517. 
General Education

AnAN National College of Technology

265, AOKI, ANAN

TOKUSHIMA 774-0017

JAPAN

E-mail address: nishimori@anan-nct.ac.jp 